\title{
The Entropy Rounding Method in Approximation Algorithms
}

\author{
Thomas Rothvoß* \\ M.I.T. \\ rothvoss@math.mit.edu
}

October 15, 2018

\begin{abstract}
Let $A$ be a matrix, $c$ be any linear objective function and $x$ be a fractional vector, say an LP solution to some discrete optimization problem. Then a recurring task in theoretical computer science (and in approximation algorithms in particular) is to obtain an integral vector $y$ such that $A x \approx A y$ and $c^{T} y$ exceeds $c^{T} x$ by only a moderate factor.

We give a new randomized rounding procedure for this task, provided that $A$ has bounded $\Delta$ approximate entropy. This property means that for uniformly chosen random signs $\chi(j) \in\{ \pm 1\}$ on any subset of the columns, the outcome $A \chi$ can be approximately described using a sub-linear number of bits in expectation.

To achieve this result, we modify well-known techniques from the field of discrepancy theory, especially we rely on Beck's entropy method, which to the best of our knowledge has never been used before in the context of approximation algorithms. Our result can be made constructive using the Bansal framework based on semidefinite programming.

We demonstrate the versatility of our procedure by rounding fractional solutions to column-based linear programs for some generalizations of BIN PACKING. For example we obtain a polynomial time $O P T+O\left(\log ^{2} O P T\right)$ approximation for BIN PACKING WITH REJECTION and the first AFPTAS for the TRAIN DELIVERY problem.
\end{abstract}

\footnotetext{
* Supported by the Alexander von Humboldt Foundation within the Feodor Lynen program.
} 


\section{Introduction}

Many approximation algorithms are based on linear programming relaxations; for the sake of concreteness, say on formulations like

$$
\min \left\{c^{T} x \mid A x \geq b, x \geq \mathbf{0}\right\},
$$

with $A \in \mathbb{R}^{n \times m}$. Several techniques have been developed to round a fractional LP solution $x$ to an integer one; the textbooks [Vaz01, WS11] provide a good overview on the most common approaches. The aim of this paper is to introduce a new LP rounding technique that we term entropy rounding.

To describe our method, we consider the random variable $A \chi$, where $\chi \in\{ \pm 1\}^{m}$ is a uniformly chosen random coloring of the columns of $A$. Suppose that $A$ has the property that one can approximately encode the outcome of $A \chi$ up to an additive error of $\Delta$ with at most $\frac{m}{5}$ bits in expectation. In other words, we suppose that we can find some arbitrary function $f$ such that $\|A \chi-f(\chi)\|_{\infty} \leq \Delta$ and the entropy of the random variables $f(\chi)$ can be bounded by $\frac{m}{5}$. Note that the entropy could never exceed $m$, hence we only need to save a constant factor by allowing an approximation error. One possible choice could be $f(\chi)=2 \Delta\left\lceil\frac{A \chi}{2 \Delta}\right\rfloor$, meaning that we round every entry of $A \chi$ to the nearest multiple of $2 \Delta$. To bound the entropy of $f(\chi)$ one can then use standard concentration bounds since the values $A_{i} \chi=\sum_{j=1}^{m} A_{i j} \chi(j)$ are the sum of independently distributed random variables (here $A_{i}$ denotes the $i$ th row of $A$ ). If this holds also for any submatrix of $A$, we say that $A$ has bounded $\Delta$-approximate entropy.

But why would it be useful to have this property for $A$ ? Since there are $2^{m}$ many colorings $\chi$, there must be an exponential number of colorings $\chi^{(1)}, \ldots, \chi^{(\ell)} \in\{ \pm 1\}^{m}$, which are similar w.r.t. $A$, i.e. $\| A \chi^{(i)}-$ $A \chi^{\left(i^{\prime}\right)} \|_{\infty} \leq \Delta$. Since there are so many similar colorings, we can pick two of them (say $\left.\chi^{(i)}, \chi^{\left(i^{\prime}\right)}\right)$ that differ in at least half of the entries and define $\chi:=\frac{1}{2}\left(\chi^{(i)}-\chi^{\left(i^{\prime}\right)}\right)$ as the difference of those colorings. Then $\chi$ is a half-coloring, i.e. it has entries in $\{-1,0,1\}$, but at least half of the entries are non-zero and furthermore $\|A \chi\|_{\infty} \leq \Delta$.

However, our aim was to find a vector $y \in\{0,1\}^{m}$ such that $A y \approx A x$. We will iteratively obtain halfcolorings and use them to update $x$, each time reducing its fractionality. Thus, we consider the least value bit in any entry of $x$; say this is bit $K$. Let $J \subseteq[m]$ be the set of indices where this bit is set to one and let $A^{J} \subseteq A$ be the submatrix of the corresponding columns. Then by the argument above, there is a half-coloring $\chi \in\{0, \pm 1\}^{J}$ such that $\left\|A^{J} \chi\right\|_{\infty} \leq \Delta$. We use this information to round our fractional solution to $x^{\prime}:=x+\left(\frac{1}{2}\right)^{K} \chi$, meaning that we delete the $K$ th bit of those entries $j$ that have $\chi(j)=-1$; we round the entry up if $\chi(j)=1$ and we leave it unchanged if $\chi(j)=0$. After iterating this at most $\log m$ times, the $K$ th bit of all entries of $x$ will be 0 . Hence after at most $K \cdot \log m$ iterations, we will end up in a $0 / 1$ vector that we term $y$. This vector satisfies $\|A x-A y\|_{\infty} \leq \sum_{k=1}^{K}\left(\frac{1}{2}\right)^{k} \cdot \log m \cdot \Delta \leq \log m \cdot \Delta$.

Let us illustrate this abstract situation with a concrete example. For the very classical BIN PACKING problem, the input consists of a sorted list of item sizes $1 \geq s_{1} \geq \ldots \geq s_{n}>0$ and the goal is to assign all items to a minimum number of bins of size 1. Let $S=\left\{S \subseteq[n] \mid \sum_{i \in S} s_{i} \leq 1\right\}$ be the set system containing all feasible patterns and let $\mathbf{1}_{S}$ denote the characteristic vector of a set $S$. A well-studied column-based LP relaxation for BIN PACKING is

$$
\min \left\{\mathbf{1}^{T} x \mid \sum_{S \in S} x_{S} \mathbf{1}_{S}=\mathbf{1}, x \geq \mathbf{0}\right\}
$$

(see e.g. Eis57, GG61, KK82]). In an integral solution, the variable $x_{S}$ tells whether a bin should be packed exactly with the items in $S$. We want to argue why our method is applicable here. Thus let $x$ be a fractional solution to (1). In order to keep the notation simple let us assume for now, that all items have size between $\frac{1}{2 k}$ and $\frac{1}{k}$. Our choice for matrix $A$ is as follows: Let $A_{i}$ be the sum of the first $i$ rows of the constraint matrix of (1), i.e. $A_{i S}=|S \cap\{1, \ldots, i\}|$. By definition, for an integral vector $y, A_{i} y$ denotes the 
number of slots that $y$ reserves for items in $1, \ldots, i$. If there are less than $i$ many slots reserved, we term this a deficit. Since we assumed that the items are sorted according to their size, a vector $y \in\{0,1\}^{S}$ will correspond to a feasible solution if there is no deficit for any interval $1, \ldots, i$.

To understand why this matrix $A$ has the needed property, we can add some artificial rows until consecutive rows differ in exactly one entry; say $n^{\prime} \leq m k$ is the new number of rows. Then observe that the sequence $A_{1} \chi, A_{2} \chi, \ldots, A_{n^{\prime}} \chi$ describes a symmetric random walk with step size 1 on the real axis. We imagine all multiples of $\Delta$ as "mile stones" and choose $f_{i}(\chi)$ as the last such mile stone that was crossed by the first $i$ steps of the random walk (i.e. by $A_{1} \chi, \ldots, A_{i} \chi$ ). For an independent random walk it would take $\Theta\left(\Delta^{2}\right)$ iterations in expectation until a random walk covers a distance of $\Delta$, thus we expect that the sequence $f_{1}(\chi), \ldots, f_{n^{\prime}}(\chi)$ changes its value only every $\Theta\left(\Delta^{2}\right)$ steps and consequently the entropy of this sequence cannot be large. But up to $k$ steps of the random walk correspond to the same column of $A$ and depend on each other. Using more involved arguments, we will still be able to show that for $\Delta:=\Theta\left(\frac{1}{k}\right)$, the entropy of the sequence $f_{1}(\chi), \ldots, f_{n^{\prime}}(\chi)$ is bounded by $\frac{m}{5}$.

More generally, we allow that the parameter $\Delta$ depends on the row $i$ of $A$. Then the same arguments go through for $\Delta_{i}:=\Theta\left(\frac{1}{s_{i}}\right)$, where $s_{i}$ is the size of item $i$. Thus our rounding procedure can be applied to a fractional BIN PACKING solution $x$ to provide an integral vector $y$ with $\left|A_{i} x-A_{i} y\right| \leq O(\log n) \cdot \Delta_{i}$. The deficits can be eliminated by buying $O\left(\log ^{2} n\right)$ extra bins in total.

The entropy-based argument which guarantees the existence of proper half-colorings $\chi$ is widely termed "Beck's Entropy Method" from the field of discrepancy theory. This area studies the discrepancy of set systems, i.e. the maximum difference of "red" and "blue" elements in any set for the best 2-coloring. Formally, the discrepancy of a set system $S \subseteq 2^{[n]}$ is defined as

$$
\operatorname{disc}(\boldsymbol{S})=\min _{\chi:[n] \rightarrow\{ \pm 1\}} \max _{S \in S}|\chi(S)| .
$$

In fact, for a variety of problems, the entropy method is the only known technique to derive the best bounds (see e.g. [Spe85, SST]).

\subsection{Related work}

Most approximation algorithms that aim at rounding a fractional solution to an integral one, use one of the following common techniques: A classical application of the properties of basic solutions yields a 2-approximation for UNRELATED MACHINE SCHEDULING [LST87]. Iterative rounding was e.g. used in a 2-approximation for a wide class of network design problems, like STEINER NETWORK [Jai98], randomized rounding can be used for a $O(\log n / \log \log n)$-approximation for MIN CONGESTION [RT87] or ATSP $\mathrm{AGM}^{+} 10$. A combination of both techniques provides the currently best approximation guarantee for STEINER TREe [BGRS10]. The dependent rounding scheme was successfully applied to LPs of an assignment type [GKPS06]. Sophisticated probabilistic techniques like the Lovász Local Lemma were for example used to obtain $O(1)$-approximation for the SANTA CLAUS problem [Fei08, HSS10].

However, to the best of our knowledge, the entropy method has never been used for the purpose of approximation algorithms, while being very popular for finding low discrepancy colorings. For the sake of comparison: for a general set system $S$ with $n$ elements, a random coloring provides an easy bound of $\operatorname{disc}(\boldsymbol{S}) \leq O(\sqrt{n \log (2|\boldsymbol{S}|)})$ (see e.g. [Mat99]). But using the Entropy method, this can be improved to $\operatorname{disc}(\boldsymbol{S}) \leq O(\sqrt{n \log (2|\boldsymbol{S}| / n)})$ for $n \leq|\boldsymbol{S}|[$ Spe85]. This bound is tight, if no more properties on the set system are specified. Other applications of this method give a $O(\sqrt{t} \log n)$ bound if no element is in more than $t$ sets [Sri97] and a $O(\sqrt{k} \log n)$ bound for the discrepancy of $k$ permutations. For the first quantity, 
alternative proof techniques give bounds of $2 t-1$ [BF81] and $O(\sqrt{t \cdot \log n})$ [Ban98]. We recommend the book of Matoušek [Mat99] (Chapter 4) for an introduction to discrepancy theory.

The entropy method itself is purely existential due to the use of the pigeonhole principle. But in a very recent breakthrough, Bansal [Ban10] showed how to obtain colorings matching the Spencer [Spe85] and Srinivasan [Sri97] bounds, by considering a random walk guided by the solution of a semidefinite program.

\section{Our contributions}

In this work, we present a very general rounding theorem which for a given vector $x \in[0,1]^{m}$, matrices $A$ and $B$, weights $\mu_{i}$ and an objective function $c$, computes a binary random vector $y$ which (1) preserves all expectations; (2) guarantees worst case bounds on $\left|A_{i} x-A_{i} y\right|$ and $\left|B_{i} x-B_{i} y\right|$ and (3) provides strong tail bounds. The bounds for $A$ depend on the entropy of random functions that approximately describe the outcomes of random colorings of subsets of columns of $A$, while the bounds for rows of $B$ are functions of the weights $\mu_{i}$.

We use this rounding theorem to obtain better approximation guarantees for several well studied BIN PACKING generalizations. In fact, so far all asymptotic FPTAS results for BIN PACKING related problems in the literature are based on rounding a basic solution to a column-based LP using its sparse support. We give the first alternative method to round such LPs, which turns out to be always at least as good as the standard technique (e.g. for classical BIN PACKING) and significantly stronger for several problems. We demonstrate this by providing the following results:

- A randomized polynomial time $O P T+O\left(\log ^{2} O P T\right)$ algorithm for BIN PACKING With ReJECTION, where in contrast to classical BIN PACKING, each item can either be packed into a bin or rejected at a given cost. Our result improves over the previously best bound of $O P T+\frac{O P T}{(\log O P T)^{1-o(1)}}[$ [EL10].

- We give the first (randomized) AFPTAS for the TRAIN DELIVERY problem, which is a combination of a one-dimensional vehicle routing problem and BIN PACKING. In fact, our algorithm produces solutions of cost $O P T+O\left(O P T^{3 / 5}\right)$ (see [DMM10] for an APTAS).

It would not be difficult to extend this list with further variants 1 , but we also believe that the method will find applications that are not related to BIN PACKING.

\section{Organization}

We recall some tools and notation in Section 2, In Section 3 we revisit results from discrepancy theory and modify them for our purposes. In Section 4 we show our general rounding theorem. Then in Sections 5 and 6 we demonstrate how our rounding theorem can be used to obtain approximation algorithms. In the Appendix we provide details on how to turn the existential proofs into polynomial time algorithms using semidefinite programming and how to solve the presented LP relaxations in polynomial time.

\footnotetext{
${ }^{1}$ Some examples: In Generalized Cost VARIABLe Size Bin Packing a list of bin types $j=1, \ldots, k$, each one with individual cost $c_{j} \in[0,1]$ and capacity $b_{j} \in[0,1]$ is given (see [EL08 for an APTAS). We can obtain a $O P T+O\left(\log ^{2} n\right)$ approximation. In its well-studied special case of VARIABLE SIZE BIN PACKING the bin costs equal the bin capacities (i.e. $c_{j}=b_{j}$ for all $j$ ) and we can refine the bound to $O P T+O\left(\log ^{2} O P T\right.$ ) (see Mur87 for an AFPTAS). For Bin PACKING With CARDinAlity ConstrainTs, no bin may receive more than $K$ items [EL09]. We can get an $O P T+O\left(\log ^{2} n\right)$ approximation. However, we postpone proofs of this claims to the full version.
} 


\section{Preliminaries}

The entropy of a random variable $Z$ is defined as

$$
H(Z)=\sum_{x} \operatorname{Pr}[Z=x] \cdot \log _{2}\left(\frac{1}{\operatorname{Pr}[Z=x]}\right)
$$

Here the sum runs over all values that $Z$ can attain. Imagine that a data source generates a string of $n$ symbols according to distribution $Z$. Then intuitively, an optimum compression needs asymptotically for $n \rightarrow \infty$ an expected number of $n \cdot H(Z)$ many bits to encode the string. Two useful facts on entropy are:

- Uniform distribution maximizes entropy: If $Z$ attains $k$ distinct values, then $H(Z)$ is maximal if $Z$ is the uniform distribution. In that case $H(Z)=\log _{2}(k)$. Conversely, if $H(Z) \leq \delta$, then there must be at least one event $x$ with $\operatorname{Pr}[Z=x] \geq\left(\frac{1}{2}\right)^{\delta}$.

- Subadditivity: If $Z, Z^{\prime}$ are random variables and $f$ is any function, then $H\left(f\left(Z, Z^{\prime}\right)\right) \leq H(Z)+H\left(Z^{\prime}\right)$.

We define $H_{\chi \in\{ \pm 1\}^{m}} f(\chi)$ as the entropy of $f(\chi)$, where $\chi$ is uniformly chosen from $\{ \pm 1\}^{m}$. See the book of [AS08] for an intensive introduction into properties of the entropy function. We will make use of the Azuma-Hoeffding Inequality (see e.g. Theorem 12.4 in [MU05]).

Lemma 1. Let $X_{1}, \ldots, X_{n}$ be random variables with $\left|X_{i}\right| \leq \alpha_{i}$ and $E\left[X_{i} \mid X_{1}, \ldots, X_{i-1}\right]=0$ for all $i=1, \ldots, n$. Let $X:=\sum_{i=1}^{n} X_{i}$. Then $\operatorname{Pr}\left[|X| \geq \lambda\|\alpha\|_{2}\right] \leq 2 e^{-\lambda^{2} / 2}$ for any $\lambda \geq 0$. This still holds, if the distribution of $X_{i}$ is an arbitrary function of $X_{1}, \ldots, X_{i-1}$.

The sequence $X_{1}, \ldots, X_{n}$ is called a Martingale and the $\alpha_{i}$ 's are the corresponding step sizes. Another tool that we are going to use is a special case of the so-called Isoperimetric Inequality of Kleitman [Kle66.

Lemma 2. For any $X \subseteq\{0,1\}^{m}$ of size $|X| \geq 2^{0.8 m}$ and $m \geq 2$, there are $x, y \in X$ with $\|x-y\|_{1} \geq m / 2$.

A function $\chi:[m] \rightarrow\{0, \pm 1\}$ is called a partial coloring. If at most half of the entries are 0 , then $\chi$ is called a half-coloring. For a quantity $z \in \mathbb{Z},\lceil z\rfloor$ denotes the integer that is closest to $z$ (say in case of a tie we round down). If $z \in \mathbb{R}^{m}$, then $\lceil z\rfloor=\left(\left\lceil z_{1}\right\rfloor, \ldots,\left\lceil z_{m}\right\rfloor\right)$. For a matrix $A \in \mathbb{R}^{n \times m}$ and $J \subseteq\{1, \ldots, m\}$, $A^{J}$ denotes the submatrix containing only the columns indexed in $J$. A submatrix $A^{\prime} \subseteq A$ will always correspond to a subset of columns of $A$, i.e. $A^{\prime} \in \mathbb{R}^{n \times m^{\prime}}$ with $m^{\prime} \leq m$. If $\chi: J \rightarrow \mathbb{R}$ is only defined on a subset $J \subseteq\{1, \ldots, m\}$ and we write $A \chi$, then we implicitly fill the undefined entries in $[m] \backslash J$ with zeros. We say that an entry $x_{i} \in\left[0,1\right.$ [ has a finite dyadic expansion with $K$ bits, if there is a sequence $b_{1}, \ldots, b_{K} \in\{0,1\}$ with $x_{i}=\sum_{k=1}^{K} 2^{-k} \cdot b_{k}$.

\section{Discrepancy theory revisited}

Initially the entropy method was developed to find a coloring $\chi:[m] \rightarrow\{ \pm 1\}$ minimizing $\left|\sum_{i \in S} \chi_{i}\right|$ for all sets in a set system, or equivalently to color columns of the incidence matrix $A \in\{0,1\}^{n \times m}$ of the set system in order to minimize $\|A \chi\|_{\infty}$. In contrast, in our setting the matrix $A$ can have arbitrary entries, but the main technique still applies. 
Theorem 3. Let $A \in \mathbb{R}^{n \times m}$ be a matrix with parameters $\Delta_{1}, \ldots, \Delta_{n}>0$ such that

$$
\underset{\bar{\chi} \in\{ \pm 1\}^{m}}{H}\left(\left\{\left[\frac{A_{i} \bar{\chi}}{2 \Delta_{i}}\right\rfloor\right\}_{i=1, \ldots, n}\right) \leq \frac{m}{5}
$$

Then there exists a half-coloring $\chi:[m] \rightarrow\{ \pm 1,0\}$ with $\left|A_{i} \chi\right| \leq \Delta_{i}$ for all $i=1, \ldots, n$.

Proof. From the assumption, we obtain that there must be a $b \in \mathbb{Z}^{n}$ such that

$$
\operatorname{Pr}_{\bar{\chi} \in\{ \pm 1\}^{m}}\left[\left\{\left\lceil\frac{A_{i} \bar{\chi}}{2 \Delta_{i}}\right]\right\}_{i=1, \ldots, n}=b\right] \geq\left(\frac{1}{2}\right)^{m / 5}
$$

In other words there is a subset $\boldsymbol{Y} \subseteq\{ \pm 1\}^{m}$ of at least $2^{m} \cdot\left(\frac{1}{2}\right)^{m / 5}=2^{\frac{4}{5} m}$ colorings such that $\left\lceil\frac{A_{i} \chi}{2 \Delta_{i}}\right\rfloor=b_{i}$ for all $\chi \in \boldsymbol{Y}$ and $i=1, \ldots, n$. The Isoperimetric Inequality (Lemma2) then yields the existence of $\chi^{\prime}, \chi^{\prime \prime} \in \boldsymbol{Y}$ with $\left|\left\{j \mid \chi_{j}^{\prime} \neq \chi_{j}^{\prime \prime}\right\}\right| \geq m / 2$. We choose $\chi_{j}:=\frac{1}{2}\left(\chi_{j}^{\prime}-\chi_{j}^{\prime \prime}\right)$, then $\chi \in\{0, \pm 1\}^{m}$ is the desired half-coloring. Finally, let us inspect the discrepancy of $\chi:\left|A_{i} \chi\right| \leq \frac{1}{2}\left|A_{i} \chi^{\prime}-A_{i} \chi^{\prime \prime}\right| \leq \Delta_{i}$.

The core of this proof was to show that there is an exponential number of colorings $\chi^{\prime}, \chi^{\prime \prime}$ that are similar, meaning that $A \chi^{\prime} \approx A \chi^{\prime \prime}$. This was done by considering disjoint intervals of length $2 \Delta_{i}$ (for every $i$ ) and using entropy to argue that many colorings must fall into the same intervals. But on the other hand, $A_{i} \chi^{\prime}$ and $A_{i} \chi^{\prime \prime}$ might be very close to each other, while they fall into different intervals and $\chi^{\prime}, \chi^{\prime \prime}$ would not count as being similar.

Hence we want to generalize the notion of similarity from Theorem 3 , Let $A \in \mathbb{R}^{n \times m}$ be a matrix and $\Delta=\left(\Delta_{1}, \ldots, \Delta_{n}\right)$ be a vector with $\Delta_{i}>0$. Then we define the $\Delta$-approximate entropy of $A$ as 2

$$
H_{\Delta}(A):=\min _{f_{1}, \ldots, f_{n}:\{ \pm 1\}^{m} \rightarrow \mathbb{R}}\left\{{ }_{\chi \in\{ \pm 1\}^{m}}^{H}\left(f_{1}(\chi), \ldots, f_{n}(\chi)\right):\left|A_{i} \chi-f_{i}(\chi)\right| \leq \Delta_{i} \forall i=1, \ldots, n\right\}
$$

First of all note that $H_{\Delta}(A)$ is always upper bounded by the entropy of the random variables $\left\lceil\frac{A_{i} \chi}{2 \Delta_{i}}\right\rfloor$, since one can choose $f_{i}(\chi):=2 \Delta_{i} \cdot\left\lceil\frac{A_{i} \chi}{2 \Delta_{i}}\right\rfloor$. On the other hand, the claim of Theorem 3 still holds true if the assumption is replaced by $H_{\Delta}(A) \leq \frac{m}{5}$, since then one has exponentially many colorings $\boldsymbol{Y}$ such that the values $f_{i}(\chi)$ coincide for every $\chi \in \boldsymbol{Y}$ and hence for every half-coloring $\chi:=\frac{1}{2}\left(\chi^{\prime}-\chi^{\prime \prime}\right)$ obtained from colorings $\chi^{\prime}, \chi^{\prime \prime} \in \boldsymbol{Y}$ one has $\left|A_{i} \chi\right| \leq \frac{1}{2}\left|\left(A_{i} \chi^{\prime}-f_{i}\left(\chi^{\prime}\right)\right)-\left(A_{i} \chi^{\prime \prime}-f_{i}\left(\chi^{\prime \prime}\right)\right)\right| \leq \Delta_{i}$. More formally:

Corollary 4. Let $A \in \mathbb{R}^{n \times m}, \Delta:=\left(\Delta_{1}, \ldots, \Delta_{n}\right)>\mathbf{0}$ with $H_{\Delta}(A) \leq \frac{m}{5}$. Then there exists a half-coloring $\chi$ : $[m] \rightarrow\{ \pm 1,0\}$ with $-\Delta \leq A \chi \leq \Delta$.

Moreover, also $H_{\Delta}$ is subadditive, i.e. $H_{\left(\Delta, \Delta^{\prime}\right)}\left(\left[\begin{array}{l}A \\ B\end{array}\right] \leq H_{\Delta}(A)+H_{\Delta^{\prime}}(B)\right.$, which follows directly from the subadditivity of the entropy function (here $\left[{ }_{B}^{A}\right]$ is obtained by stacking matrices $A$ and $B$ ).

For now let us consider a concrete method of bounding the entropy of a random variable of the form $\left\lceil\frac{\alpha^{T} \chi}{2 \Delta}\right\rfloor$, where $\alpha$ is one of the row vectors of $A$. Recall that this immediately upperbounds $H_{\Delta}(\alpha)$. For this purpose, we again slightly adapt a lemma from discrepancy theory (see e.g. Chapter 4 in [Mat99]).

\footnotetext{
${ }^{2}$ The minimum is always attained since all probabilities are multiplies of $\left(\frac{1}{2}\right)^{m}$ and consequently the entropy can attain only a finite number of values.
} 
Lemma 5. Let $\alpha \in \mathbb{R}^{m}$ be a vector and $\Delta>0$. For $\lambda=\frac{\Delta}{\|\alpha\|_{2}}$,

$$
\underset{\chi \in\{ \pm 1\}^{m}}{H}\left(\left\lceil\frac{\alpha^{T} \chi}{2 \Delta}\right\rfloor\right) \leq G(\lambda):= \begin{cases}9 e^{-\lambda^{2} / 5} & \text { if } \lambda \geq 2 \\ \log _{2}(32+64 / \lambda) & \text { if } \lambda<2\end{cases}
$$

The proof can be found in Appendix $\left[\right.$, But the intuition is as follows: Abbreviate $Z:=\left\lceil\frac{\alpha^{T} \chi}{2 \Delta}\right\rfloor$. Then $\operatorname{Pr}[Z=0] \geq 1-e^{-\Omega\left(\lambda^{2}\right)}$ and $\operatorname{Pr}[Z=i] \leq e^{-\Omega\left(i^{2} \lambda^{2}\right)}$ for $i \neq 0$. A simple calculation yields that $H(Z) \leq e^{-\Omega\left(\lambda^{2}\right)}$. But for $\lambda \ll 2$, with high probability one has at least $|Z| \leq O\left(\frac{1}{\lambda}\right)$ and consequently $H(Z) \leq \log O\left(\frac{1}{\lambda}\right)$.

The following function $G^{-1}(b)$ will denote the discrepancy bound $\Delta$ that we need to impose, if we do not want to account an entropy contribution of more than $b$.

$$
G^{-1}(b):= \begin{cases}\sqrt{10 \ln \left(\frac{9}{b}\right)} & 0<b \leq 6 \\ 128 \cdot\left(\frac{1}{2}\right)^{b} & b>6\end{cases}
$$

Strictly spoken, $G^{-1}$ is not the inverse of $G$, but it is not difficult to verify that $G\left(G^{-1}(b)\right) \leq b$ for all $b>0$. In other words, for any vector $\alpha$ and value $b>0$, we can choose $\Delta:=G^{-1}(b) \cdot\|\alpha\|_{2}$, then $H\left(\left\lceil\frac{\alpha^{T} \chi}{2 \Delta}\right]\right) \leq b$.

\section{The main theorem}

Now we have all ingredients for our main theorem, in which we iteratively round a fractional vector $x$ using half-colorings $\chi$. Concerning the choice of parameters $\Delta$, one has in principle two options: One can either give static bounds $\Delta_{i}$ to rows $A_{i}$ such that $H_{\Delta}\left(A^{\prime}\right) \leq \frac{\# \operatorname{col}\left(A^{\prime}\right)}{5}$ holds for any submatrix $A^{\prime} \subseteq A$; or one can assign a fixed fraction to each row and then letting $\Delta_{i}$ be a function of $\# \operatorname{col}\left(A^{\prime}\right)$. In fact, we will combine these approaches, which will turn out to be useful later.

Theorem 6. Assume the following is given: A matrix $A \in \mathbb{R}^{n_{A} \times m}$, parameters $\Delta=\left(\Delta_{1}, \ldots, \Delta_{n_{A}}\right)>\mathbf{0}$ such that $\forall J \subseteq\{1, \ldots, m\}: H_{\Delta}\left(A^{J}\right) \leq \frac{|J|}{10}$, a matrix $B \in[-1,1]^{n_{B} \times m}$, weights $\mu_{1}, \ldots, \mu_{n_{B}}>0$ with $\sum_{i=1}^{n_{B}} \mu_{i} \leq 1$, a vector $x \in[0,1]^{m}$ and an objective function $c \in[-1,1]^{m}$. Then there is a random variable $y \in\{0,1\}^{m}$ with

- Preserved expectation: $E\left[c^{T} y\right]=c^{T} x, E[A y]=A x, E[B y]=B x$.

- Bounded difference: $\left|c^{T} x-c^{T} y\right| \leq O(1) ;\left|A_{i} x-A_{i} y\right| \leq \log (\min \{4 n, 4 m\}) \cdot \Delta_{i}$ for all $i=1, \ldots, n_{A}(n:=$ $\left.n_{A}+n_{B}\right) ;\left|B_{i} x-B_{i} y\right| \leq O\left(\sqrt{1 / \mu_{i}}\right)$ for all $i=1, \ldots, n_{B}$.

- Tail bounds: $\forall i: \forall \lambda \geq 0: \operatorname{Pr}\left[\left|A_{i} x-A_{i} y\right| \geq \lambda \cdot \sqrt{\log (\min \{4 n, 4 m\})} \cdot \Delta_{i}\right] \leq 2 e^{-\lambda^{2} / 2}$.

Proof. First, observe that we can append the objective function as an additional row to matrix $B$ (with a weight of say $\mu_{c}:=\frac{1}{2}$ and halving the other $\mu_{i}$ 's), and so we ignore it from now on. Next, consider the linear system

$$
\begin{aligned}
A z & =A x \\
B z & =B x \\
0 \leq z_{j} & \leq 1 \quad \forall j=1, \ldots, m
\end{aligned}
$$

and let $z$ be a basic solution. Apart from the $0 / 1$ bounds, the system has only $n$ constraints, hence the number of entries $j$ with $0<z_{j}<1$ is bounded by $n$. One can remove columns of $A$ with $z_{j} \in\{0,1\}$ and apply the Theorem to the residual instance. Hence we set $x:=z$ and assume from now on that $m \leq n$. 
Furthermore we assume that $x$ has a finite dyadic expansion, i.e. every entry $x_{j}$ it can be written in binary encoding with $K$ bits, for some $K \in \mathbb{N}$. This can be achieved by randomly rounding the entries of $x$ to either the nearest larger or smaller multiple of $\left(\frac{1}{2}\right)^{K}$ for a polynomially large $K$, while the error is exponentially small in $K{ }^{3}$ We perform the following rounding procedure:

(1) WHILE $x$ not integral DO

(2) Let $k \in\{1, \ldots, K\}$ be the index of the least value bit in any entry of $x$

(3) $J:=\left\{j \in\{1, \ldots, m\} \mid x_{j}\right.$ 's $k$ th bit is 1$\}$

(4) Choose $\chi \in\{0, \pm 1\}^{m}$ with $\chi(j)=0$ for $j \notin J$, $|\operatorname{supp}(\chi)| \geq|J| / 2,\left|A_{i} \chi\right| \leq \Delta_{i}$ and $\left|B_{i} \chi\right| \leq G^{-1}\left(\mu_{i}|J| / 10\right)$. $\sqrt{|J|}$ for all $i$.

(5) With probability $\frac{1}{2}$, flip all signs in $\chi$

(6) Update $x:=x+\left(\frac{1}{2}\right)^{k} \chi$

The interval of iterations in which bit $k$ is rounded, is termed phase $k$. Let $x^{(k)}$ be the value of $x$ at the beginning of phase $k$ and let $x^{(k, t)}$ denote the value of $x$ at the beginning of the $t$ th to last iteration of phase $k$. From now on $x$ always denotes the initial value, i.e. $x=x^{(K)}$ and our choice for the rounded vector is $y:=x^{(0)}$.

Observe that flipping the signs in step (5) ensures that the expectations are preserved, i.e. $E[A y]=A x$ and $E[B y]=B x$. There are two main issues: $(I)$ showing that the choice of $\chi$ in step (4) is always possible; $(I I)$ bounding the rounding error of $y$ w.r.t. $x$.

Claim (I). For any $J \subseteq\{1, \ldots, m\}$ there is a $\chi \in\{0, \pm 1\}^{m}$ with $\chi(j)=0$ for $j \notin J,|\operatorname{supp}(\chi)| \geq|J| / 2,\left|A_{i} \chi\right| \leq \Delta_{i}$ and $\left|B_{i} \chi\right| \leq G^{-1}\left(\frac{\mu_{i}|J|}{10}\right) \cdot \sqrt{|J|}$ for all $i$.

Proof of claim. Our aim is to apply Theorem 3 to the stacked $n \times|J|$ matrix $\tilde{A}=\left[\begin{array}{l}A^{J} \\ B^{j}\end{array}\right]$ with parameter $\tilde{\Delta}:=$ $\left(\Delta, \Delta^{\prime}\right)$ and $\Delta_{i}^{\prime}:=G^{-1}\left(\frac{\mu_{i}|J|}{10}\right) \cdot \sqrt{|J|}$. Note that $\left\|B_{i}^{J}\right\|_{2} \leq \sqrt{|J|}$ since $B$ has entries in $[-1,1]$, hence the entropy that we need to account to the $i$ th row of $B$ is $H\left(\left[\frac{B_{i} \chi}{2 \Delta_{i}^{\prime}}\right]\right) \leq \frac{\mu_{i}|J|}{10}$. By subadditivity of the (approximate) entropy function and the assumption that $H_{\Delta}\left(A^{J}\right) \leq \frac{|J|}{10}$,

$$
H_{\tilde{\Delta}}(\tilde{A}) \leq H_{\Delta}\left(A^{J}\right)+\sum_{i=1}^{n_{B}} \underset{\chi \in\{ \pm 1\}^{J}}{H}\left(\left\lceil\frac{B_{i}^{J} \chi}{2 \Delta_{i}^{\prime}}\right\rfloor\right) \leq \frac{|J|}{10}+\sum_{i=1}^{n_{B}} \frac{\mu_{i}|J|}{10} \leq \frac{|J|}{5} .
$$

Thus the requirements of Theorem 3 are met, which then implies the existence of the desired halfcoloring and Claim $(I)$ follows.

The next step is to bound the rounding error.

Claim (II). One has $\left|A_{i} x-A_{i} y\right| \leq \log (2 m) \cdot \Delta_{i}$ for all $i=1, \ldots, n_{A}$ and $\left|B_{i} x-B_{i} y\right| \leq O\left(\sqrt{1 / \mu_{i}}\right)$ for all $i=$ $1, \ldots, n_{B}$.

Proof of claim. Let $J(k, t)=\left\{j \mid x_{j}^{(k, t)}\right.$, s $k$ th bit is 1$\}$ denote the set $J$ in the $t$ th to last iteration of phase $k$, i.e. $J(k, t) \supset J(k, t-1)$ for any $t$. Since the cardinality of $J(k, t)$ drops by a factor of at least $1 / 2$ from

\footnotetext{
${ }^{3}$ Note that we have the term $\min \{4 m, 4 n\}$ instead of $\min \{2 m, 2 n\}$ in the claim, to account for the rounding error to obtain a vector $x$ with dyadic expansion and to account for the extra row $c$ that we appended to $B$.
} 
iteration to iteration, we have $|J(k, t-1)| \leq \frac{1}{2} \cdot|J(k, t)|$ for any $t$. Hence each phase has at $\operatorname{most} \log _{2}(m)+1$ iterations. Then for any $i=1, \ldots, n_{A}$

$$
\left|A_{i} y-A_{i} x\right| \leq\left|\sum_{k=1}^{K} \sum_{t \geq 0} A_{i}\left(x^{(k, t)}-x^{(k, t+1)}\right)\right| \leq \sum_{k=1}^{K} \log (2 m) \cdot\left(\frac{1}{2}\right)^{k} \Delta_{i} \leq \log (2 m) \cdot \Delta_{i}
$$

using that $\left|A_{i}\left(x^{(k, t)}-x^{(k, t+1)}\right)\right| \leq\left(\frac{1}{2}\right)^{k} \Delta_{i}$ and $\sum_{k \geq 1}\left(\frac{1}{2}\right)^{k}=1$. Next, consider

$$
\begin{aligned}
& \left|B_{i} x-B_{i} y\right| \leq \sum_{k=1}^{K}\left(\frac{1}{2}\right)^{k} \sum_{t \geq 0} G^{-1}\left(\frac{\mu_{i}|J(k, t)|}{10}\right) \cdot \sqrt{|J(k, t)|} \\
& \stackrel{(*)}{\leq} \quad \sum_{z \in \mathbb{Z}} G^{-1}\left(6 \cdot 2^{z}\right) \cdot \sqrt{2^{z+1} \frac{60}{\mu_{i}}} \\
& \underset{\leq}{\operatorname{Def} G^{-1}} \sqrt{\frac{120}{\mu_{i}}}\left[\sum_{z \geq 0} 128\left(\frac{1}{2}\right)^{2^{z}} 2^{z / 2}+\sum_{z \geq 0} \sqrt{10 \ln \left(\frac{9}{6} 2^{z}\right)} \cdot\left(\frac{1}{2}\right)^{z / 2}\right] \\
& \stackrel{(* *)}{\leq} \quad O\left(\sqrt{1 / \mu_{i}}\right)
\end{aligned}
$$

In $(*)$ we use that since $|J(k, t+1)| \geq 2 \cdot|J(k, t)|$, for any $k$ and $z$, there is at most one $t$ such that $6 \cdot 2^{z} \leq$ $\frac{\mu_{i}|J(k, t)|}{10}<6 \cdot 2^{z+1} ;(* *)$ follows from the convergence of $\sum_{z \geq 0}(1 / 2)^{2^{z}-z / 2}$ and $\sum_{z \geq 0} \sqrt{z \cdot(1 / 2)^{z}}$.

Inspecting (2) again, we see that $A_{i} x-A_{i} y$ is a Martingale and the step size in iteration $t \leq \log (2 m)$ of phase $k$ is bounded by $\alpha_{k, t}:=\left(\frac{1}{2}\right)^{k} \Delta_{i}$. Observe that $\|\alpha\|_{2} \leq \Delta_{i} \sqrt{\log _{2}(2 m)}$, hence the tail bound $\operatorname{Pr}\left[\mid A_{i} x-\right.$ $\left.A_{i} y \mid \geq \lambda \cdot \sqrt{\log (2 m)} \cdot \Delta_{i}\right] \leq 2 e^{-\lambda^{2} / 2}$ for all $\lambda \geq 0$ follows from the Azuma-Hoeffding Inequality (Lemma(1). This concludes the proof of the theorem.

Moreover, we can also compute such a vector $y$ as guaranteed by the theorem in polynomial time, with the only exception that the guaranteed bound on $\left|B_{i} x-B_{i} y\right|$ is slightly weaker. But we still can provide that $E\left[\left|B_{i} x-B_{i} y\right|\right]=O\left(\sqrt{1 / \mu_{i}}\right)$, which is already sufficient for our applications. We postpone the algorithmic details to Appendix $\mathrm{A}$.

For one example application, let $B \in\{0,1\}^{n \times n}$ be the incidence matrix of a set system with $n$ sets on a ground set of $n$ elements. Then apply Theorem6 with $A=\mathbf{0}, x=\left(\frac{1}{2}, \ldots, \frac{1}{2}\right)$ and $\mu_{i}=\frac{1}{n}$ to obtain a $y \in\{0,1\}^{n}$ with $\|B x-B y\|_{\infty}=O(\sqrt{n})$. The coloring $\chi \in\{0,1\}^{n}$ with $y=x+\frac{1}{2} \chi$ is then an $O(\sqrt{n})$ discrepancy coloring, matching the bound of Spencer [Spe85]. Note that no proof using a different technique is known for Spencer's theorem. Hence it seems unlikely that Theorem 6 (in particular the dependence on $1 / \mu_{i}$ ) could be achieved by standard techniques (such as using properties of basic solutions or the usual independent randomized rounding).

\section{Application: Bin Packing with Rejection}

For classical BIN PACKING, the input consists of a list of item sizes $1 \geq s_{1} \geq \ldots \geq s_{n}>0$ and the goal is to assign the items to a minimum number of bins of size 1. For the performance of heuristics like First Fit, Next Fit and First Fit Decreasing, see [Joh73, JDU ${ }^{+} 74$, CGJ84]. A proof of strong NP-hardness can be found in [GJ79]. Fernandez de la Vega and Luecker [FdIVL81 developed an asymptotic polynomial time approximation scheme (APTAS). Later, Karmarkar and Karp [KK82] (see also [KV02, WS11]) found an algorithm that needs at most $O\left(\log ^{2} O P T\right)$ bins more than the optimum solution. 
In this section, we provide an application of our Entropy Rounding Theorem to the more general problem of Bin PACKIng With Rejection, where every item $i$ can either be packed into a unit cost bin or it can be rejected at cost $\pi_{i}>0$ (which is also part of the input). The first constant factor approximation and online algorithms were studied in [DH06]. Later an asymptotic PTAS was developed by [Eps06, Eps10] (see [BCH08] for a faster APTAS). Recently Epstein \& Levin [EL10] found an algorithm with running time polynomial in the input length and $\frac{1}{\varepsilon}$ which provides solutions of quality $(1+\varepsilon) O P T+$ $2^{O\left(\frac{1}{\varepsilon} \log \frac{1}{\varepsilon}\right)}$ (implying an $A P X \leq O P T+\frac{O P T}{(\log O P T)^{1-o(1)}}$ algorithm for an optimum choice of $\varepsilon$ ).

An asymptotic FPTAS (AFPTAS) is defined as an approximation algorithm APX producing solutions with $A P X \leq(1+\varepsilon) O P T+f(1 / \varepsilon)$ in polynomial time (both in the input length and $\frac{1}{\varepsilon}$ ). But there is some ambiguity in the literature what concerns the term $f(1 / \varepsilon)$. According to [KV02] and [ZMO07], $f$ can be any function (equivalent to the requirement $A P X \leq O P T+o(O P T)$ ), while Johnson [Joh85] requires $f$ to be bounded by a polynomial (which is equivalent to $A P X \leq O P T+O\left(O P T^{1-\delta}\right.$ ) for a fixed $\delta>0$ ). However, we will now obtain a polynomial time algorithm for BIN PACKING WITH REJECTION with $A P X \leq O P T+O\left(\log ^{2} O P T\right)$, which satisfies also the stronger definition of Johnson [Joh85] and matches the bound for the special case of BIN PACKING (without rejection).

We define a set system $\boldsymbol{S}=\boldsymbol{B} \cup \boldsymbol{R}$ with potential bin patterns $\boldsymbol{B}=\left\{S \subseteq[n] \mid \sum_{i \in S} s_{i} \leq 1\right\}$ (each set $S \in \boldsymbol{B}$ has cost $\left.c_{S}:=1\right)$ and rejections $\boldsymbol{R}=\{\{i\} \mid i \in[n]\}$ at $\operatorname{cost} c_{\{i\}}:=\pi_{i}$ for $i \in[n]$. Then a natural column-based $\mathrm{LP}$ is

$$
O P T_{f}=\min \left\{c^{T} x \mid \sum_{S \in S} x_{S} \mathbf{1}_{S}=\mathbf{1}, x \geq \mathbf{0}\right\}
$$

where $\mathbf{1}_{S} \in\{0,1\}^{n}$ denotes the characteristic vector of $S$. In [EKRS11], the Karmarkar-Karp technique [KK82] was modified to obtain a $O\left(\sqrt{n} \cdot \log ^{3 / 2} n\right)$ bound on the additive integrality gap of (3). Note that due to the dependence on $n$, such a bound does not satisfy the definition of an AFPTAS, and hence is incomparable to the result of [EL09]. But since $O P T_{f} \leq n$, our result improves over both bounds [EL09, EKRS11].

Despite the exponential number of variables in LP (3), one can compute a basic solution $x$ with $c^{T} x \leq O P T_{f}+\delta$ in time polynomial in $n$ and $1 / \delta$ [KK82] using either the Grötschel-Lovász-Schrijver variant of the Ellipsoid method [GLS81] or the Plotkin-Shmoys-Tardos framework for covering and packing problems [PST95]. Since this fact is rather standard, we postpone details to Appendix B.

In the following we always assume that the items are sorted w.r.t. their sizes such that $s_{1} \geq \ldots \geq s_{n}$ and $\pi_{i} \leq 1$ for all $i=1, \ldots, n$. A feasible solution $y \in\{0,1\}^{S}$ will reserve at least one slot for every item, i.e. $i$ many slots for items $1, \ldots, i$. The quantity $i-\sum_{S \in S} y_{S}|S \cap\{1, \ldots, i\}|$, if positive, is called the deficit of $\{1, \ldots, i\}$. It is not difficult to see that if there is no deficit for any of the sets $\{1, \ldots, i\}$, then every item can be assigned to a slot - potentially of a larger item 4 (while in case that $y_{S}=1$ for $S \in \boldsymbol{R}$, the slot for $S=\{i\}$ would only be used for that particular item).

We term the constraint matrix $P$ of the system (3) the pattern matrix. Note that some columns of $P$ correspond to bins, others correspond to rejections. The obvious idea would be to apply our rounding theorem to $P$, but this would not yield any reasonable bound. Instead, we define another matrix $A$ of the same format as $P$, where $A_{i}:=\sum_{i^{\prime}=1}^{i} P_{i^{\prime}}$ or equivalently, the entries are defined as $A_{i S}=|S \cap\{1, \ldots, i\}|$. The intuition behind this is that if $x$ is a feasible fractional solution then $A y-A x \geq \mathbf{0}$ iff $y$ does not have any deficit. Indeed, we will apply Theorem 6 to this cumulated pattern matrix $A$. As a prerequisite, we need a strong upper bound on the approximate entropy of any submatrix.

\footnotetext{
${ }^{4}$ Proof sketch: Assign input items $i$ iteratively in increasing order (starting with the largest one, i.e. $i=1$ ) to the smallest available slot. If there is none left for item $i$, then there are less then $i$ slots for items $1, \ldots, i$, thus this interval had a deficit.
} 
Lemma 7. Let $A \in \mathbb{Z}_{\geq 0}^{n \times m}$ be any matrix in which column $j$ has non-decreasing entries from $\left\{0, \ldots, b_{j}\right\}$; let $\sigma=\sum_{j=1}^{m} b_{j}$ be the sum over the largest entries in each column and $\beta:=\max _{j=1, \ldots, m} b_{j}$ be the maximum of those entries. For $\Delta>0$ one has

$$
H_{\Delta}(A) \leq O\left(\frac{\sigma \beta}{\Delta^{2}}\right)
$$

Proof. We can add rows (and delete identical rows) such that consecutive rows differ in exactly one entry. This can never lower the approximate entropy. Now we have exactly $n=\sigma$ many rows. There is no harm in assuming 5 that $\sigma, \Delta$ and $\beta$ are powers of 2 . Let $B \in \mathbb{R}^{\sigma \times m}$ be the matrix with $B_{i}=A_{i}-A_{i-1}$ (and $B_{1}=A_{1}$ ). In other words, $B$ is a $0 / 1$ matrix with exactly a single one per row.

Consider the balanced binary laminar dissection $D:=\left\{\left\{2^{k}(i-1)+1, \ldots, 2^{k} i\right\} \mid k=0, \ldots, \log \sigma ; i=\right.$ $\left.1, \ldots, \frac{\sigma}{2^{k}}\right\}$ of the row indices $\{1, \ldots, \sigma\}$. In other words, $D$ contains $2^{k}$ many intervals of length $\sigma / 2^{k}$ for $k=0, \ldots, \log _{2} \sigma$. For every of those interval $D \in D$ we define the vector $C_{D}:=\sum_{i \in D} B_{i} \in \mathbb{Z}^{m}$ and parameter $\Delta_{D}:=\frac{\Delta}{32 \cdot 1.1^{|z|}}$ if $|D|=2^{z} \cdot \frac{\Delta^{2}}{\beta}$ (with $z \in \mathbb{Z}$ ). Note that since $B$ contains a single one per row and at most $\beta$ ones per column, thus $\left\|C_{D}\right\|_{2} \leq\left(\left\|C_{D}\right\|_{\infty} \cdot\left\|C_{D}\right\|_{1}\right)^{1 / 2} \leq \sqrt{\beta \cdot|D|}=2^{z / 2} \cdot \Delta 6$

For every $i$, note that $\{1, \ldots, i\}$ can be written as a disjoint union of some intervals $D_{1}, \ldots, D_{q} \in \boldsymbol{D}$, all of different size. We choose $f_{i}(\chi):=\sum_{p=1}^{q} 2 \Delta_{D_{p}} \cdot\left\lceil\frac{C_{D_{p}} \chi}{2 \Delta_{D_{p}}} \mid\right.$. Then

$$
\left|A_{i} \chi-f_{i}(\chi)\right| \leq \sum_{p=1}^{q} \Delta_{D_{p}} \leq \sum_{z \in \mathbb{Z}} \frac{\Delta}{32 \cdot 1.1^{|z|}} \leq \Delta .
$$

Thus $H_{\Delta}(A)$ is upper bounded by the entropy of the random variables $f_{1}(\chi), \ldots, f_{n}(\chi)$. But since each $f_{i}$ is a function of $\left\{\left[C_{D} \chi /\left(2 \Delta_{D}\right)\right]\right\}_{D \in D}$, it in fact suffices to bound the entropy of the latter random variables. We remember that for all $z \in \mathbb{Z}$, one has at most $\frac{\sigma \beta}{2^{z} \Delta^{2}}$ many intervals $D \in \boldsymbol{D}$ of size $|D|=\frac{2^{z} \Delta^{2}}{\beta}$, with $\left\|C_{D}\right\|_{2} \leq 2^{z / 2} \cdot \Delta$ and $\Delta_{D}=\frac{\Delta}{32 \cdot 1.1^{|z|}} \geq \frac{\Delta}{32 \cdot 1.1^{|z|} \cdot \Delta 2^{z / 2}}\left\|C_{D}\right\|_{2} \geq \frac{1}{32 \cdot 1.3^{z}}\left\|C_{D}\right\|_{2}$. Finally

$$
\begin{aligned}
& \underset{\chi \in\{ \pm 1\}^{m}}{H}\left(\left\{\left\lceil\frac{C_{D} \chi}{2 \Delta_{D}}\right\rfloor\right\}_{D \in D}\right) \stackrel{\substack{H \text { subadd. } \\
\text { \&Lem.5 }}}{\leq} \sum_{z \in \mathbb{Z}} \frac{\sigma \beta}{2^{z} \Delta^{2}} \cdot G\left(\frac{1}{32 \cdot 1.3^{z}}\right)
\end{aligned}
$$

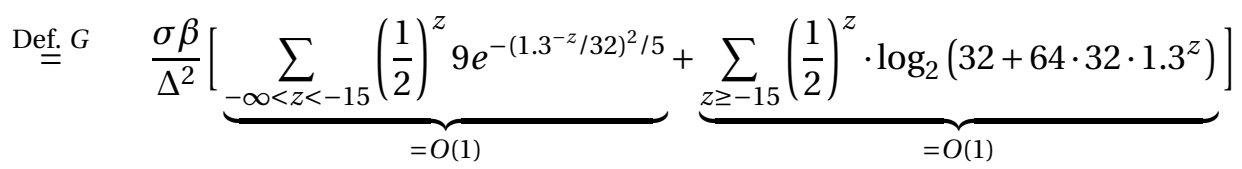

$$
\begin{aligned}
& =\quad O\left(\frac{\sigma \beta}{\Delta^{2}}\right)
\end{aligned}
$$

The parametrization used in the above proof is inspired by the work of Spencer, Srinivasan and Tetali [SST]. A simple consequence of the previous lemma is the following.

Lemma 8. Let $S_{1}, \ldots, S_{m} \subseteq[n]$ be a set system with numbers $1 \geq s_{1} \geq \ldots \geq s_{n}>0$ such that $\sum_{i \in S_{j}} s_{i} \leq 1$ for any set $S_{j}$. Let $A \in \mathbb{Z}_{\geq 0}^{n \times m}$ be the cumulated pattern matrix, defined by $A_{i j}=\left|S_{j} \cap\{1, \ldots, i\}\right|$. Then there is a constant $C>0$ such that for $\Delta:=\frac{C}{s_{i}}$, one has $H_{\Delta}(A) \leq \frac{1}{10} \sum_{j=1}^{m} \sum_{i \in S_{j}} s_{i} \leq \frac{m}{10}$.

\footnotetext{
${ }^{5}$ In fact, rounding $\sigma, \beta, \Delta$ to the nearest power of 2 only affect the constant hidden in the $O(1)$-notation.

${ }^{6}$ Here we use Hölder's inequality: $\|x\|_{2} \leq\left(\|x\|_{\infty} \cdot\|x\|_{1}\right)^{1 / 2}$ for every $x \in \mathbb{R}^{m}$.
} 
Proof. Let $A^{\ell}$ be the submatrix of $A$ consisting of all rows $i$ such that $s_{i} \geq(1 / 2)^{\ell}$. Note that row $A_{i}$ possibly appears in several $A^{\ell}$. We apply Lemma 7 to $A^{\ell}$ with $\beta:=\left\|A^{\ell}\right\|_{\infty} \leq 2^{\ell}$ and $\sigma:=\sum_{j=1}^{m}\left|S_{j} \cap\left\{i: s_{i} \geq(1 / 2)^{\ell}\right\}\right|$ to obtain $H_{C \cdot 2^{\ell}}\left(A^{\ell}\right) \leq \frac{1}{20} \sum_{j=1}^{m}(1 / 2)^{\ell} \cdot\left|S_{j} \cap\left\{i: s_{i} \geq(1 / 2)^{\ell}\right\}\right|$ for $C$ large enough. Eventually

$$
H_{\Delta}(A) \stackrel{\text { subadd. }}{\leq} \sum_{\ell \geq 0} H_{C \cdot 2^{\ell}}\left(A^{\ell}\right) \leq \frac{1}{20} \sum_{\ell \geq 0} \sum_{j=1}^{m}(1 / 2)^{\ell} \cdot\left|S_{j} \cap\left\{i: s_{i} \geq(1 / 2)^{\ell}\right\}\right| \leq \frac{1}{10} \sum_{j=1}^{m} \sum_{i \in S_{j}} s_{i}
$$

since item $i \in S_{j}$ with $(1 / 2)^{\ell} \leq s_{i}<(1 / 2)^{\ell+1}$ contributes at most $\frac{1}{20} \sum_{\ell^{\prime} \geq \ell}(1 / 2)^{\ell^{\prime}} \leq \frac{1}{10} s_{i}$ to the left hand side.

Our procedure to round a fractional BIN PACKING WiTH REJECTION solution $x$ will work as follows: For a suitable value of $\varepsilon>0$, we term all items of size at least $\varepsilon$ large and small otherwise. We take the cumulated pattern matrix $A$ restricted to the large items. Furthermore we define a matrix $B$ such that $B x$ denotes the space reserved for small items. Then we apply Theorem 6 to obtain an integral vector $y$ which is then repaired to a feasible solution without significantly increasing the cost of the solution.

Theorem 9. There is a randomized algorithm for BIN PACKING WITH REJECTION with expected polynomial running time which produces a solution of cost $O P T_{f}+O\left(\log ^{2} O P T_{f}\right)$.

Proof. Compute a fractional solution $x \in[0,1]^{S}$ to the Bin PACKing With Rejection LP (3) with cost $c^{T} x \leq O P T_{f}+1$ (see Appendix $B$ for details). We define $\varepsilon:=\frac{\log \left(O P T_{f}\right)}{O P T_{f}}$ (assume $O P T_{f} \geq 2$ ). For any item $i$ that is rejected in $x$ to a fractional extend of more than $1-\varepsilon$ (i.e. $x_{\{i\}}>1-\varepsilon$ ), we fully reject item $i$. We account a multiplicative loss of $1 /(1-\varepsilon) \leq 1+2 \varepsilon$ (i.e. an additive cost increase of $O\left(\log O P T_{f}\right)$ ). From now on, we may assume that $x_{S} \leq 1-\varepsilon$ for all sets $S \in \boldsymbol{R}$. Let $1, \ldots, L$ be the items of size at least $\varepsilon$, hence $O P T_{f} \geq \varepsilon^{2} L$, since every item is covered with bin patterns at an extend of at least $\varepsilon$. Let $A \in \mathbb{Z}^{L \times S}$ with

$$
A_{i S}:=|\{1, \ldots, i\} \cap S|
$$

be the cumulated pattern matrix restricted to the large items. According to Lemma 8 , for a choice of $\Delta_{i}=\Theta\left(1 / s_{i}\right)$, one has $H_{\Delta}\left(A^{\prime}\right) \leq \frac{\# \operatorname{col}\left(A^{\prime}\right)}{10}$ for every submatrix $A^{\prime} \subseteq A$. Choose $B \in[0,1]^{1 \times S}$ as the row vector where $B_{1, S}=\sum_{i \in S: i>L} s_{i}$ for $S \in S$ denotes the space in pattern $S$ that is reserved for small items.

We apply Theorem 16 (Theorem 6 suffices for a non-constructive bound on the integrality gap) to matrices $A, B$ and cost function $c$ with $\mu_{1}=1$ to obtain a vector $y \in\{0,1\} S$ with the following properties

(A) The deficit of any interval $\{1, \ldots, i\}$ of large items (i.e. $i \in\{1, \ldots, L\})$ is bounded by $O\left(\frac{1}{s_{i}} \log L\right)$.

(B) The space for small items reserved by $y$ equals that of $x$ up to an additive constant term (formally $\left.\left|\sum_{S \in S}\left(x_{S}-y_{S}\right) \cdot B_{1, S}\right|=O(1)\right)$.

(C) $c^{T} y \leq c^{T} x+O(1)$

For $\ell \geq 0$, we say that the items $G^{\ell}:=\left\{i \leq L \mid\left(\frac{1}{2}\right)^{\ell} \geq i>\left(\frac{1}{2}\right)^{\ell+1}\right\}$ form group $\ell$. Note that at most $\frac{1}{\varepsilon}+$ 1 groups contain large items. We eliminate the deficits for large items by packing $O(\log L)$ extra bins with the largest item from every group, hence leading to $O(\log L) \cdot O\left(\log \frac{1}{\varepsilon}\right)=O\left(\log ^{2} O P T_{f}\right)$ extra bins. Property $(B)$ implies that after buying $O(1)$ extra bins for small items, it is possible to assign all small items fractionally to the bought bins (i.e. the small items could be feasibly assigned if it would be allowed to split them). By a standard argument (see e.g. [EL09]) this fractional assignment can be turned into an integral assignment, if we discard at most one item per pattern, i.e. we pack discarded small items of total size at most $\varepsilon \cdot\left(c^{T} y+O(1)\right)$ separately, which can be done with $O(\varepsilon) \cdot O P T_{f}+O(1) \leq O\left(\log O P T_{f}\right)$ extra bins. The claim follows. 


\section{Application: The Train Delivery Problem}

For the TRAIN DELIVERY problem, $n$ items are given as input, but now every item $i \in\{1, \ldots, n\}$ has a size $s_{i}$ and a position $p_{i} \in[0,1]$. The goal is to transport the items to a depot, located at 0 , using trains of capacity 1 and minimizing the total tour length 7 . In other words, it is a combination of one-dimensional vehicle routing and BIN PACKING. We define $S$ as a set system consisting of all sets $S \subseteq[n]$ with $\sum_{i \in S} s_{i} \leq 1$ and cost $c_{S}:=\max _{i \in S} p_{i}$ for set $S$. Then the optimum value for Train DeliverY equals the cost of the cheapest subset of $S$ covering all items (ignoring a constant factor of two).

The problem was studied in [DMM10], where the authors provide an APTAS. We will now obtain an AFPTAS. First, we make our life easier by using a result of [DMM10] saying that modulo a $1+O(\varepsilon)$ factor in the approximation guarantee it suffices to solve well-rounded instances which have the property that $\varepsilon \leq p_{i} \leq 1$ and $p_{i} \in(1+\varepsilon)^{\mathbb{Z}}$ for all $i=1, \ldots, n$. The first condition can be obtained by splitting all tours in a proper way; the second condition is obtained by simply rounding all positions up to the nearest power of $1+\varepsilon$. Hence, we can partition the items according to their position by letting $P_{j}:=\left\{i \mid p_{i}=(1+\varepsilon)^{j}\right\}$ for $j=0, \ldots, t-1$ with $t=O\left(\frac{1}{\varepsilon} \log \frac{1}{\varepsilon}\right)$.

Our rounding procedure works as follows: analogously to BIN PACKING WITH REJECTION, we construct matrices $A(j), B(j)$ separately for the items at each position $j$. Then we stack them together; apply Theorem 6 , and repair the obtained integral vector to a feasible solution (again analogous to the previous section). Here we will spend a higher weight $\mu_{j}$ for positions $j$ which are further away from the depot since those are costlier to cover.

Theorem 10. There is a randomized algorithm with expected polynomial running time for TRAIN DELIVERY, providing solutions of expected cost $E[A P X] \leq O P T_{f}+O\left(O P T_{f}^{3 / 5}\right)$.

Proof. Compute a fractional solution $x$ for the TRAIN DELIVERY LP (i.e. again LP (3), but with the problem specific set system and cost vector) of $\operatorname{cost} c^{T} x \leq O P T_{f}+1$ (see Appendix B for details). We will choose $\varepsilon:=1 / O P T_{f}^{\delta}$ for some constant $0<\delta<1$ that we determine later and assume the instance is well-rounded.

By $1, \ldots, L$ we denote the large items of size $\geq \varepsilon$. Let $A(j) \in \mathbb{Z}^{\left(P_{j} \cap[L]\right) \times S}$ with entries $A_{i, S}(j)=\mid S \cap$ $\{1, \ldots, i\} \cap P_{j} \mid$ be the cumulated pattern matrix, restricted to large items at position $P_{j}$. We equip again every row $A_{i}(j)$ with parameter $\Delta_{i}(j):=\Theta\left(1 / s_{i}\right)$. Then we stack $A(0), \ldots, A(t-1)$ together to obtain an $L \times|\boldsymbol{S}|$ matrix $A$. Again, we need to show that for any submatrix $A^{\prime} \subseteq A$, one has $H_{\Delta}\left(A^{\prime}\right) \leq \frac{\# \operatorname{col}\left(A^{\prime}\right)}{10}$. Let $\boldsymbol{S}^{\prime} \subseteq \boldsymbol{S}$ be the sets whose characteristic vectors form the columns of $A^{\prime}$. We apply Lemma 8 individually to each $A^{\prime}(j)$ and obtain

$$
H_{\Delta}\left(A^{\prime}\right) \leq \sum_{j=0}^{t-1} H_{\Delta(j)}\left(A^{\prime}(j)\right) \leq \frac{1}{10} \sum_{j=0}^{t-1} \sum_{S \in S^{\prime}} \sum_{i \in S \cap P_{j}} s_{i} \leq \frac{\# \operatorname{col}\left(A^{\prime}\right)}{10}
$$

using that every set $S$ contains items of total size at most 1 . Furthermore, we define a matrix $B \in[0,1]^{t \times S}$ with $B_{j, S}:=\sum_{i \in S \cap P_{j}: i>L} s_{i}$ as the space that pattern $S$ reserves for small items at position $j$. We equip the $j$ th row of $B$ with weight $\mu_{j}:=\frac{\varepsilon}{5} \cdot(1+\varepsilon / 4)^{-j}$, i.e. the weight grows with the distance to the depot. Note that $\sum_{j \geq 0} \mu_{j} \leq \sum_{j \geq 0} \frac{\varepsilon}{5} \cdot(1+\varepsilon / 4)^{-j}=\frac{4}{5}+\frac{\varepsilon}{5} \leq 1$. Moreover $O P T_{f} \geq \sum_{i \in[n]} s_{i} p_{i} \geq \varepsilon^{2} \cdot L$ (see [DMM10]), hence the number of rows of $A$ and $B$ is $L+t \leq \operatorname{poly}\left(O P T_{f}\right)$.

\footnotetext{
${ }^{7}$ For definiteness, say the tour must start and end at the depot and once items are loaded into the train, they have to remain until the depot is reached.
} 
We apply Theorem 16 (again Theorem 6 suffices for an integrality gap bound) to obtain an integral vector $y \in\{0,1\}^{S}$ with the following error guarantees:

- Large items: Let $i \in P_{j}, i \leq L$. Then the deficit of $\{1, \ldots, i\} \cap P_{j}$ is bounded by $O\left(\frac{1}{s_{i}} \log O P T_{f}\right)$.

For every position we use $O\left(\log ^{2} O P T_{f}\right)$ extra bins to eliminate the deficits of large items, which costs in total $\sum_{j \geq 0}(1+\varepsilon)^{-j} \cdot O\left(\log ^{2} O P T_{f}\right)=O\left(\frac{1}{\varepsilon} \log ^{2} O P T_{f}\right)$.

- Small items: For position $j$, the expected discrepancy in the reserved space for small items is $E\left[\left|B_{j} x-B_{j} y\right|\right] \leq O\left(\sqrt{1 / \mu_{j}}\right)=O\left(\sqrt{1 / \varepsilon \cdot(1+\varepsilon / 4)^{j}}\right)$.

We buy $\left|B_{j} x-B_{j} y\right|$ extra bins to cover small items at position $j$. Their expected cost is bounded by $O(\sqrt{1 / \varepsilon}) \cdot\left(1+\frac{\varepsilon}{4}\right)^{j / 2} \cdot(1+\varepsilon)^{-j} \leq O(\sqrt{1 / \varepsilon}) \cdot(1-\varepsilon / 2)^{j}$. In total, this accounts with an expected cost increase of $O(\sqrt{1 / \varepsilon}) \sum_{j \geq 0}(1-\varepsilon / 2)^{j}=O(1) \cdot(1 / \varepsilon)^{3 / 2}$ for all positions. Now, for every position the space reserved for small items is at least as large as the required space, hence the small items can be assigned fractionally. Then after discarding at most one small item per pattern, even an integral assignment is possible. We account this with a multiplicative factor of $1 /(1-\varepsilon) \leq 1+2 \varepsilon$.

Summing up the bought extra bins, we obtain a solution $A P X$ with

$$
E[A P X] \leq(1+O(\varepsilon)) O P T_{f}+O\left(\frac{1}{\varepsilon} \log ^{2} O P T_{f}\right)+O\left((1 / \varepsilon)^{3 / 2}\right) \leq O P T_{f}+O\left(O P T_{f}^{3 / 5}\right) .
$$

$\operatorname{choosing} \varepsilon:=O P T_{f}^{-2 / 5}$.

Acknowledgments. The author is grateful to Michel X. Goemans, Neil Olver, Rico Zenklusen, Laura Sanità and Nikhil Bansal for helpful advice and remarks. Thanks to Saurabh Ray for marketing advice.

\section{References}

$\left[\mathrm{AGM}^{+} 10\right]$ A. Asadpour, M. X. Goemans, A. Madry, S. O. Gharan, and A. Saberi. An O $(\log \mathrm{n} / \log \log \mathrm{n})-$ approximation algorithm for the asymmetric traveling salesman problem. In Moses Charikar, editor, SODA, pages 379-389. SIAM, 2010.

[AS08] N. Alon and J. H. Spencer. The probabilistic method. Wiley-Interscience Series in Discrete Mathematics and Optimization. John Wiley \& Sons Inc., Hoboken, NJ, third edition, 2008. With an appendix on the life and work of Paul Erdős.

[Ban98] W. Banaszczyk. Balancing vectors and Gaussian measures of $n$-dimensional convex bodies. Random Structures Algorithms, 12(4):351-360, 1998.

[Ban10] N. Bansal. Constructive algorithms for discrepancy minimization. In FOCS, pages 3-10, 2010.

[BCH08] W. Bein, J. R. Correa, and X. Han. A fast asymptotic approximation scheme for bin packing with rejection. Theoret. Comput. Sci., 393(1-3):14-22, 2008.

[BF81] J. Beck and T. Fiala. "Integer-making” theorems. Discrete Appl. Math., 3(1):1-8, 1981.

[BGRS10] J. Byrka, F. Grandoni, T. Rothvoß, and L. Sanità. An improved LP-based approximation for Steiner tree. In Leonard J. Schulman, editor, STOC, pages 583-592. ACM, 2010. 
[CGJ84] E. G. Coffman, Jr., M. R. Garey, and D. S. Johnson. Approximation algorithms for binpacking-an updated survey. In Algorithm design for computer system design, volume 284 of CISM Courses and Lectures, pages 49-106. Springer, Vienna, 1984.

[DH06] G. Dósa and Y. He. Bin packing problems with rejection penalties and their dual problems. Inf. Comput., 204:795-815, May 2006.

[DMM10] A. Das, C. Mathieu, and S. Mozes. The train delivery problem- vehicle routing meets bin packing. In 8th Workshop on Approximation and Online Algorithms (WAOA'10), pages 94105, 2010.

[Eis57] K. Eisemann. The trim problem. Management Science, 3(3):279-284, 1957.

[EKRS11] F. Eisenbrand, N. Kakimura, T. Rothvoß, and L. Sanità. Set covering with ordered replacement - additive and multiplicative gaps. In Proceedings of the 15th International Conference on Integer Programming and Combinatorial Optimization, IPCO 2011, 2011.

[EL08] L. Epstein and A. Levin. An APTAS for generalized cost variable-sized bin packing. SIAM Journal on Computing, 38(1):411-428, 2008.

[EL09] L. Epstein and A. Levin. AFPTAS results for common variants of bin packing: A new method to handle the small items. CoRR, abs/0906.5050, 2009.

[EL10] L. Epstein and A. Levin. AFPTAS results for common variants of bin packing: A new method for handling the small items. SIAM Journal on Optimization, 20(6):3121-3145, 2010.

[Eps06] L. Epstein. Bin packing with rejection revisited. In Approximation and online algorithms, volume 4368 of Lecture Notes in Comput. Sci., pages 146-159. Springer, Berlin, 2006.

[Eps10] L. Epstein. Bin packing with rejection revisited. Algorithmica, 56(4):505-528, 2010.

[FdlVL81] W. Fernandez de la Vega and G. S. Lueker. Bin packing can be solved within $1+\varepsilon$ in linear time. Combinatorica, 1(4):349-355, 1981.

[Fei08] U. Feige. On allocations that maximize fairness. In Shang-Hua Teng, editor, SODA, pages 287-293. SIAM, 2008.

[GG61] P. C. Gilmore and R. E. Gomory. A linear programming approach to the cutting-stock problem. Operations Research, 9:849-859, 1961.

[GJ79] M. R. Garey and D. S. Johnson. Computers and Intractability: A Guide to the Theory of NPCompleteness. W. H. Freeman and Company, New York, New York, 1979.

[GKPS06] R. Gandhi, S. Khuller, S. Parthasarathy, and A. Srinivasan. Dependent rounding and its applications to approximation algorithms. J. ACM, 53(3):324-360, 2006.

[GLS81] M. Grötschel, L. Lovász, and A. Schrijver. The ellipsoid method and its consequences in combinatorial optimization. Combinatorica, 1(2):169-197, 1981.

[Goe97] M. X. Goemans. Semidefinite programming in combinatorial optimization. Mathematical Programming, 79:143-161, 1997. 
[GW04] M. X. Goemans and D. P. Williamson. Approximation algorithms for MAX-3-CUT and other problems via complex semidefinite programming. J. Comput. System Sci., 68(2):442-470, 2004.

[HSS10] B. Haeupler, B. Saha, and A. Srinivasan. New constructive aspects of the lovasz local lemma. CoRR, abs/1001.1231, 2010.

[Jai98] K. Jain. A factor 2 approximation algorithm for the generalized Steiner network problem. In IEEE Symposium on Foundations of Computer Science (FOCS), pages 448-457, 1998.

[JDU $\left.{ }^{+} 74\right]$ D. S. Johnson, A. Demers, J. D. Ullman, M. R. Garey, and R. L. Graham. Worst-case performance bounds for simple one-dimensional packing algorithms. SIAM Journal on Computing, 3(4):299-325, 1974.

[Joh73] D. S. Johnson. Near-optimal bin packing algorithms. PhD thesis, MIT, Cambridge, MA, 1973.

[Joh85] David S. Johnson. The np-completeness column: an ongoing guide. Journal of Algorithms, 6:434-451, 1985.

[KK82] N. Karmarkar and R. M. Karp. An efficient approximation scheme for the one-dimensional bin-packing problem. In 23rd annual symposium on foundations of computer science (Chicago, Ill., 1982), pages 312-320. IEEE, New York, 1982.

[Kle66] D. Kleitman. On a combinatorial problem of Erdős. Proc. Amer. Math. Soc., 17:139-141, 1966.

[KMS98] D. Karger, R. Motwani, and M. Sudan. Approximate graph coloring by semidefinite programming. Journal of the ACM, 45(2):246-265, 1998.

[KV02] B. Korte and J. Vygen. Combinatorial Optimization - Theory and Algorithms. Springer-Verlag, Second Edition, 2002.

[Lov03] L. Lovász. Semidefinite programs and combinatorial optimization. In Bruce A. Reed and Cláudia L. Sales, editors, Recent Advances in Algorithms and Combinatorics, volume 11 of CMS Books in Mathematics, pages 137-194. Springer-Verlag, Berlin-Heidelberg-New YorkHong Kong-London-Milan-Paris-Tokyo, 2003.

[LST87] J. K. Lenstra, D. B. Shmoys, and E. Tardos. Approximation algorithms for scheduling unrelated parallel machines. In Ashok K. Chandra, editor, Proceedings of the 28th Annual Symposium on Foundations of Computer Science, pages 217-224, Los Angeles, CA, October 1987. IEEE Computer Society Press.

[Mat99] J. Matoušek. Geometric discrepancy, volume 18 of Algorithms and Combinatorics. SpringerVerlag, Berlin, 1999. An illustrated guide.

[MU05] M. Mitzenmacher and E. Upfal. Probability and computing. Cambridge University Press, Cambridge, 2005. Randomized algorithms and probabilistic analysis.

[Mur87] Frank D. Murgolo. An efficient approximation scheme for variable-sized bin packing. SIAM J. Comput., 16:149-161, February 1987. 
[PST95] Serge A. Plotkin, David B. Shmoys, and Éva Tardos. Fast approximation algorithms for fractional packing and covering problems. Math. Oper. Res., 20(2):257-301, 1995.

[Ram95] M. Ramana. An exact duality theory for semidefinite programming and its complexity implications. Mathematical Programming, 77, 1995.

[RT87] P. Raghavan and C. D. Thompson. Randomized rounding: a technique for provably good algorithms and algorithmic proofs. Combinatorica, 7(4):365-374, 1987.

[Spe85] J. Spencer. Six standard deviations suffice. Transactions of the American Mathematical Society, 289(2):679-706, 1985.

[Sri97] A. Srinivasan. Improving the discrepancy bound for sparse matrices: better approximations for sparse lattice approximation problems. In Proceedings of the Eighth Annual ACM-SIAM Symposium on Discrete Algorithms (New Orleans, LA, 1997), pages 692-701, New York, 1997. ACM.

[SST] J. H. Spencer, A. Srinivasan, and P. Tetali. The discrepancy of permutation families. Unpublished manuscript.

[Vaz01] V. Vazirani. Approximation algorithms. Springer-Verlag, Berlin, 2001.

[WS11] D. Williamson and D. Shmoys. The Design of Approximation Algorithms. Cambridge University Press, 2011.

[ZMO07] A. Zhu, R. Motwani, and L. O'Callaghan. Asymptotic Polynomial-Time Approximation Schemes. Chapman and Hall/CRC, 2011/02/28 2007. 


\section{Appendix}

\section{A Computing low-discrepancy colorings by SDP}

Observe that the only non-constructive ingredient we used is the application of the pigeonhole principle (with an exponential number of pigeons and pigeonholes) in Theorem 3 and Corollary 4 to obtain the existence of low-discrepancy half-colorings. The purpose of this section is to make this claim constructive. More precisely, we will replace each phase of $\log m$ iteratively found half-colorings by finding a single full coloring.

Theorem 11. Let $A \in \mathbb{Q}^{n_{A} \times m}, B \in[-1,1]^{n_{B} \times m}, \Delta_{1}, \ldots, \Delta_{n_{A}}>0, \mu_{1}, \ldots, \mu_{n_{B}}>0$ (with $\sum_{i} \mu_{i} \leq 1, m \geq 2$ ) be given as input. Assume that $\forall A^{\prime} \subseteq A: H_{\Delta}\left(A^{\prime}\right) \leq \frac{\# \operatorname{col}\left(A^{\prime}\right)}{10}$. Then there is a constant $C>0$ and a randomized algorithm with expected polynomial running time, which computes a coloring $\chi:[\mathrm{m}] \rightarrow\{ \pm 1\}$ such that for all $\lambda \geq 0$,

$$
\begin{aligned}
\operatorname{Pr}\left[\left|A_{i} \chi\right|>\lambda \cdot C \sqrt{\log m} \cdot \Delta_{i}\right] & \leq 4 e^{-\lambda^{2} / 2} \quad \forall i=1, \ldots, n_{A} \\
\operatorname{Pr}\left[\left|B_{i} \chi\right|>\lambda \cdot C \sqrt{1 / \mu_{i}}\right] & \leq 160 \cdot 2^{-\lambda / 6} \quad \forall i=1, \ldots, n_{B} .
\end{aligned}
$$

Note that $\Delta_{i} \geq\left\|A_{i}\right\|_{\infty}$, thus we may rescale $A$ and $\Delta$ so that $\Delta_{i} \geq 1$ and $\|A\|_{\infty} \leq 1$. We may assume that $n \geq m$. Furthermore we assume $\lambda \geq 1$ and $m$ is large enough, since otherwise all probabilities exceed 1 for $C$ suitable large and there is nothing to show. The following approach to prove Theorem 11 is a adaptation of the seminal work of Bansal [Ban10].

\section{A.1 Some preliminaries}

The Gaussian distribution $N\left(\mu, \sigma^{2}\right)$ with mean $\mu$ and variance $\sigma^{2}$ is defined by the density function

$$
f(x)=\frac{1}{\sqrt{2 \pi} \sigma} e^{-(x-\mu)^{2} /\left(2 \sigma^{2}\right)}
$$

If $g$ is drawn from this distribution, we write $g \sim N\left(\mu, \sigma^{2}\right)$. The $n$-dimensional Gaussian distribution $N^{n}(0,1)$ is obtained by sampling every coordinate $g_{i}$ independently from $N(0,1)$. Since $N^{n}(0,1)$ is rotationally symmetric, one has

Fact 12. Let $v \in \mathbb{R}^{n}$ be any vector and $g \sim N^{n}(0,1)$, then $g^{T} v \sim N\left(0,\|v\|_{2}^{2}\right)$.

\section{Martingales \& concentration bounds}

A Martingale is a sequence $0=X_{0}, X_{1}, \ldots, X_{n}$ of random variables with the property that the increment $Y_{i}:=X_{i}-X_{i-1}$ has mean $E\left[Y_{i}\right]=0$. Here $Y_{i}:=Y_{i}\left(X_{0}, \ldots, X_{i-1}\right)$ is allowed to arbitrarily depend on the previous events $X_{0}, \ldots, X_{i-1}$. We will make use of the following concentration bound:

Lemma $13\left([\overline{\text { Ban10] }})\right.$. Let $0=X_{0}, \ldots, X_{n}$ be a Martingale with increments $Y_{i}$, where $Y_{i}=\eta_{i} G_{i}, G_{i} \sim N(0,1)$ and $\left|\eta_{i}\right| \leq \delta$. Then for any $\lambda \geq 0$

$$
\operatorname{Pr}\left[\left|X_{n}\right| \geq \lambda \delta \sqrt{n}\right] \leq 2 e^{-\lambda^{2} / 2}
$$




\section{Semidefinite programming}

A matrix $Y \in \mathbb{R}^{n \times n}$ is termed positive-semidefinite (abbreviated by $Y \geq 0$ ), if $x^{T} Y x \geq 0$ for all $x \in \mathbb{R}^{n}$ (or equivalently all eigenvalues of $Y$ are non-negative). Let $S_{n}=\left\{Y \in \mathbb{R}^{n \times n} \mid Y^{T}=Y ; Y \geq 0\right\}$ be the convex cone of symmetric positive-semidefinite matrices. A semidefinite program is of the form

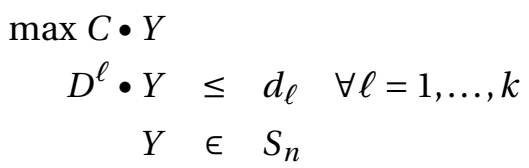

Here $C \bullet Y=\sum_{i=1}^{n} \sum_{j=1}^{n} C_{i j} \cdot Y_{i j}$ is the "vector product for matrices" (also called Frobenius product). In contrast to linear programs, it is possible that the only feasible solution to an SDP is irrational even if the input is integral. Furthermore the norm even of the smallest feasible solution might be doublyexponential in the input length Ram95]. Nevertheless, given an error parameter $\varepsilon>0$ and a ball of radius $R$ that contains at least one optimum solution (of value $S D P$ ), one can compute a $Y \in S_{n}$ with $C \bullet Y \geq S D P-\varepsilon$ and $D^{\ell} \bullet Y \leq d_{\ell}+\varepsilon$ for all $\ell=1, \ldots, k$ in time polynomial in the input length and in $\log (\max \{1 / \varepsilon, R\})$. Since for our algorithm, numerical errors could be easily absorbed into the discrepancy bounds, we always assume we have exact solutions. The first use of SDPs in approximation algorithms was the MAXCUT algorithm of Goemans and Williamson [GW04. Later on SDPs were used for example to approximate graph colorings [KMS98]. We refer to the surveys of [Lov03, Goe97] for more details on semidefinite programming.

Using that any symmetric, positive semidefinite matrix $Y$ can be written as $W^{T} W$ for $W \in \mathbb{R}^{n \times n}$ (and vice versa), the above SDP is equivalent to a vector program

$$
\begin{aligned}
& \max \sum_{i=1}^{n} \sum_{j=1}^{n} C_{i j} v_{i} v_{j} \\
& \sum_{i=1}^{n} \sum_{j=1}^{n} D_{i j}^{\ell} v_{i} v_{j} \leq d_{\ell} \quad \forall \ell=1, \ldots, k \\
& v_{i} \in \mathbb{R}^{n} \quad \forall i=1, \ldots, n
\end{aligned}
$$

\section{A.2 The algorithm}

Consider the following semidefinite program 8

$$
\begin{aligned}
\left\|\sum_{j=1}^{m} A_{i j} \cdot v_{j}\right\|_{2} & \leq \Delta_{i} \quad \forall i=1, \ldots, n_{A} \\
\left\|\sum_{j=1}^{m} B_{i j} \cdot v_{j}\right\|_{2} & \leq G^{-1}\left(\frac{\mu_{i}}{10}\left|J_{t-1}\right|\right) \cdot \sqrt{\left|J_{t-1}\right|} \quad \forall i=1, \ldots, n_{B} \\
\sum_{j=1}^{m}\left\|v_{j}\right\|_{2}^{2} & \geq \frac{\left|J_{t-1}\right|}{2} \\
\left\|v_{j}\right\|_{2} & \leq 1 \quad \forall j \in J_{t-1} \\
v_{j} & =\mathbf{0} \quad \forall j \notin J_{t-1} \\
v_{j} & \in \mathbb{R}^{m} \quad \forall j=1, \ldots, m
\end{aligned}
$$

\footnotetext{
${ }^{8}$ More precisely this program is equivalent to a semidefinite program.
} 
Here, $J_{t-1} \subseteq[m]$ denotes the set of active variables at the beginning of step $t$. Initially we have a fractional coloring $\chi^{0}=(0, \ldots, 0)$ and all variables are active, i.e. $J_{0}=[m]$. For a certain number of iterations, we sample an increment $\gamma_{t}$ using a solution from the SDP and add it to $\chi$. If a variable $\chi(j)$ reaches +1 or -1 , then we freeze it (the variable becomes inactive and is removed from $J_{t}$ ). Note that the proof of Theorem 6 guarantees that no matter which variables are currently contained in $J_{t}$, the SDP is always feasible.

Let $s:=\frac{1}{n^{2} \sqrt{8 \log (n m)}}$ be a step size and $\ell=20 \cdot \frac{16}{s^{2}} \log m$ be the number of iterations. For $t=1, \ldots, \ell$ repeat the following:

(1) Compute a solution $\left\{v_{j}\right\}_{j \in[m]}$ to the SDP

(2) Sample $g \sim N^{m}(0,1)$

(3) Update $\gamma_{t}(j):=s \cdot g^{T} v_{j}, \chi^{t}:=\chi^{t-1}+\gamma_{t}$

(4) If $\chi^{t}(j) \in\left[1-\frac{1}{n^{2}}, 1\right]\left(\left[-1,-1+\frac{1}{n^{2}}\right]\right.$, resp.) then $\chi^{t}(j):=1(-1$, resp.), $j$ becomes inactive

Note that $s \cdot g^{T} v_{j} \sim N\left(0, s^{2}\left\|v_{j}\right\|_{2}^{2}\right)$, hence $\operatorname{Pr}\left[\gamma_{t}(j)>\frac{1}{n^{2}}\right] \leq 2 \cdot e^{-(\sqrt{8 \ln (n m)})^{2} / 2}=\frac{2}{n^{4} m^{4}}$ using Lemma13, hence we may assume that $\left|\chi^{t}(j)\right|$ never exceeds 1 . No constraint $i$ will ever suffer an extra discrepancy of more than $n \cdot \frac{1}{n^{2}} \ll \Delta_{i}$ in Step (4), hence we ignore it from now on.

It was proven in [Ban10], that with high probability, after the last iteration all variables are inactive, i.e. $\chi^{\ell} \in\{ \pm 1\}^{m}$.

Lemma 14 ([Ban10]). The probability that within $16 / s^{2}$ iterations, the number of active sets decreases by a factor of at least 2 is at least $1 / 2$.

Intuitively the reason is the following: consider a variable $\chi^{t}(j)$ and suppose for simplicity that always $\left\|v_{j}\right\|_{2}=1$. Then, the values $\chi^{0}(j), \chi^{1}(j), \ldots$ behave essentially like an unbiased random walk in which in every step we go either $s$ units to the left or to the right. In a block of $\frac{1}{s^{2}}$ steps, with a constant probability we deviate $\frac{1}{s}$ steps from 0 , i.e. $\left|\chi^{t}(j)\right| \geq s \cdot \frac{1}{s}=1$ and $j$ got frozen at some point. Hence the chance that any of the $m$ variables $j$ is not frozen after $20 \log m$ blocks (each of $\frac{16}{s^{2}}$ iterations) can be easily bounded by $e^{-10 \log m \cdot 0.9^{2} / 3} \leq \frac{1}{m^{2}}$ using the Chernov bound (e.g. Thm 4.4 in [MU05).

It remains to bound the discrepancy of $\chi^{\ell}$. Let $\left\{v_{j}^{t}\right\}_{j \in[m]}$ be the SDP solution and $g_{t}$ be the random Gaussian vector in step $t$. Then

$$
A_{i} \chi^{\ell}=\sum_{t=1}^{\ell} A_{i} \gamma_{t}=\sum_{t=1}^{\ell} \sum_{j=1}^{m} s A_{i j} g_{t}^{T} v_{j}^{t}=\sum_{t=1}^{\ell} g_{t}^{T} s\left(\sum_{j=1}^{m} A_{i j} v_{j}^{t}\right)
$$

But $s \sum_{j=1}^{m} A_{i j} v_{j}^{t}$ is a vector of length at most $s \cdot \Delta_{i}$, thus $A_{i} \cdot \chi^{\ell}$ is a martingale and we can apply Lemma13 with $\delta:=s \cdot \Delta_{i}$ to bound

$$
\operatorname{Pr}\left[\left|A_{i} \chi^{\ell}\right|>\lambda \cdot \sqrt{\frac{320}{s^{2}} \log m} \cdot s \cdot \Delta_{i}\right] \leq 2 e^{-\lambda^{2} / 2} .
$$

However, we need to be a bit more careful to analyze the behavior of $\left|B_{i} \chi^{\ell}\right|$. In the following, we fix any index $i \in\left\{1, \ldots, n_{B}\right\}$. The difficulty is that the discrepancy that we allow for row $i$ changes dynamically as the number of active variables decreases. The sequence of iterations $T_{r}:=\left\{t\left|2^{r} \leq\right| J_{t-1} \mid<2^{r+1}\right\}$, in which the number of active variables is between $2^{r}$ and $2^{r+1}$ is termed phase $r$. Let $\delta(r):=G^{-1}\left(\frac{\mu_{i}}{10} 2^{r}\right) \cdot 2^{(r+1) / 2}$ 
be an upper bound on the discrepancy bound that is imposed to row $i$ during this phase. Again we can write

$$
\left|B_{i} \chi^{\ell}\right|=\left|\sum_{t=1}^{\ell} B_{i} \gamma_{t}\right|=\sum_{t=1}^{\ell}\left|g_{t}^{T} s\left(\sum_{j=1}^{m} B_{i j} v_{j}^{t}\right)\right|=\sum_{r \geq 0} \overbrace{|s \sum_{t \in T_{r}} g_{t}^{T} \underbrace{\left(\sum_{j=1}^{m} B_{i j} v_{j}^{t}\right)}_{=: u^{t}}|}^{=: X(r)}
$$

and $\left\|u^{t}\right\|_{2} \leq \delta(r)$. By $X(r)$ we denote the discrepancy that we suffer in phase $r$. We somewhat expect that $E[X(r)]=O(\delta(r))$. In fact, this is true even with a strong tail bound.

Lemma 15. For all $r \geq 0$ and $\lambda \geq 0, \operatorname{Pr}[X(r)>\lambda \cdot \delta(r)] \leq 3 \cdot 2^{-\lambda / 6}$.

Proof. If we suffer a large discrepancy in a single phase, then either the phase lasted much longer than $O\left(\frac{1}{s^{2}}\right)$ iterations or the phase was short but the discrepancy exceeded the standard deviation by a large factor. However both is unlikely. More formally, for the event $X(r)>\lambda \cdot \delta(r)$ to happen, at least one of the following events must occur

- $E_{1}:\left|T_{r}\right| \geq \frac{\lambda}{4} \cdot \frac{16}{s^{2}}$

- $E_{2}$ : Within the first $\frac{\lambda}{4} \cdot \frac{16}{s^{2}}$ iterations of phase $r$, a discrepancy of $\lambda \cdot \delta(r)$ is reached.

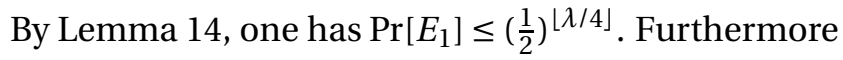

$$
\operatorname{Pr}\left[E_{2}\right] \leq \operatorname{Pr}[\left|\sum_{\text {first } \frac{4 \lambda}{s^{2}} \text { it. } t \in T_{r}} s \cdot g_{t}^{T} u^{t}\right|>\underbrace{\frac{\sqrt{\lambda}}{2} \cdot s \delta(r) \cdot \sqrt{\frac{4 \lambda}{s^{2}}}}_{=\lambda \cdot \delta(r)}] \leq 2 e^{-\lambda / 8}
$$

by again applying Lemma 13 with parameters $\lambda^{\prime}:=\frac{\sqrt{\lambda}}{2} ; \delta^{\prime}:=s \cdot \delta(r) ; n^{\prime}:=\frac{4 \lambda}{s^{2}}$. The claim follows since $\operatorname{Pr}[X(r)>\lambda \cdot \delta(r)] \leq \operatorname{Pr}\left[E_{1}\right]+\operatorname{Pr}\left[E_{2}\right] \leq 2^{-\lfloor\lambda / 4\rfloor}+2 e^{-\lambda / 8} \leq 4 \cdot 2^{-\lambda / 6}$.

By the union bound, we could easily bound the probability that any phase $r$ has $X(r)>\lambda \cdot \delta(r)$ by $4 \cdot 2^{-\lambda / 6} \cdot \log m$ and thus $\operatorname{Pr}\left[\left|B_{i} \chi^{\ell}\right|>\lambda \cdot C / \sqrt{\mu_{i}}\right] \leq 4 \cdot 2^{-\lambda / 6} \cdot \log m$. However, we can avoid the $\log m$ term by observing that the bound on $\left|B_{i} x-B_{i} y\right|$ in the proof of Theorem 6 receives the largest contributions within the small window, when the number of active variables is $\Theta\left(1 / \mu_{i}\right)$. Outside of this window, we have a lot of slack, that we can use here.

We call a phase $r$ bad, if $|X(r)|>\delta(r) \cdot\left(\lambda+\left|r-\log \left(\frac{60}{\mu_{i}}\right)\right|\right)$ (and good otherwise). Note that the term $\left|r-\log \left(\frac{60}{\mu_{i}}\right)\right|$ is indeed minimized if $2^{r}=\Theta\left(\frac{1}{\mu_{i}}\right)$. Then we can upper bound the probability that any phase is bad by

$$
\sum_{r \geq 0} \operatorname{Pr}\left[X(r)>\delta(r) \cdot\left(\lambda+\left|r-\log \left(\frac{60}{\mu_{i}}\right)\right|\right)\right] \stackrel{\text { Lem. 15 }}{\leq} 2 \sum_{z \geq 0} 4 \cdot 2^{-(\lambda+z) / 6} \leq 80 \cdot 2^{-\lambda / 6}
$$

It remains to prove that if all phases $r$ are good, then $\left|B_{i} \chi\right| \leq \lambda \cdot O\left(\sqrt{1 / \mu_{i}}\right)$. Hence we consider

$$
\begin{aligned}
\left|B_{i} \chi\right| & \stackrel{(*)}{\leq} \quad \sum_{r \geq 0} G^{-1}\left(\frac{\mu_{i} 2^{r}}{10}\right) \cdot \sqrt{2^{r+1}} \cdot\left(\lambda+\left|r-\log \left(\frac{60}{\mu_{i}}\right)\right|\right) \\
& \stackrel{(* *)}{\leq} \quad \sqrt{\frac{120}{\mu_{i}}} \sum_{z \in \mathbb{Z}} G^{-1}\left(6 \cdot 2^{z}\right) \cdot 2^{z / 2}(\lambda+|z|) \\
& \left.\stackrel{(* * *)}{\leq} \lambda \cdot C \sqrt{1 / \mu_{i}}\right)
\end{aligned}
$$


for some constant $C>0$. In (*) we assumed that all phases were good and in $(* *)$ we substitute $z:=$ $r-\log _{2}\left(\frac{60}{\mu_{i}}\right.$ ) (or equivalently $2^{z}=2^{r} \cdot \frac{\mu_{i}}{60}$ ). Eventually we recall that already in the proof of Theorem 6 we saw that the series $\sum_{z \in \mathbb{Z}} G^{-1}\left(6 \cdot 2^{z}\right) \cdot 2^{z / 2}$ converges geometrically, which is not affected by adding a polynomial term like $|z|$, hence giving $(* * *)$ (here we also use $\lambda \geq 1$ ). This almost concludes the proof of Theorem 11] since with probability at most $\frac{1}{m^{2}}+\frac{2}{n^{4} m^{4}} \cdot \ell \leq \frac{1}{2}$ (for $m$ large enough) the algorithm produces a failure, i.e. not all variables are frozen after $O\left(\frac{1}{s^{2}} \log m\right)$ iterations. In this case, we simply repeat the algorithm until it was successful. Then the actual tail bound that we obtain is a conditional probability

$$
\operatorname{Pr}\left[\left|B_{i} \chi\right|>\lambda C / \sqrt{\mu_{i}} \mid \text { run successful }\right] \leq \frac{\operatorname{Pr}\left[\left|B_{i} \chi\right|>\lambda C / \sqrt{\mu_{i}}\right]}{\operatorname{Pr}[\text { run successful }]} \leq 2 \cdot 80 \cdot 2^{-\lambda / 6}
$$

(same for $\left|A_{i} \chi\right|$ ) and the expected running time is polynomial.

\section{A.3 The Constructive Rounding Theorem}

Now that we can compute efficiently full colorings $\chi$ such that $A \chi, B \chi \approx \mathbf{0}$, it is not difficult anymore to give an algorithmic version of our main theorem.

Theorem 16. Let $A \in \mathbb{Q}^{n_{A} \times m}, B \in[-1,1]^{n_{B} \times m}, \Delta=\left(\Delta_{1}, \ldots, \Delta_{n_{A}}\right)>\mathbf{0}, \mu_{1}, \ldots, \mu_{n_{B}}>0\left(\sum_{i=1}^{n_{B}} \mu_{i} \leq 1, m \geq 2\right)$ and $c \in[-1,1]^{m}$ be given as input, such that $\forall J \subseteq\{1, \ldots, m\}: H_{\Delta}\left(A^{J}\right) \leq \frac{|J|}{10}$. Then there is a constant $C^{\prime}>0$ and a randomized algorithm with expected polynomial running time which obtains a $y \in\{0,1\}^{m}$ such that

- Preserved expectation: $E\left[c^{T} y\right]=c^{T} x, E[A y]=A x, E[B y]=B x$.

- Bounded difference: $\left|c^{T} x-c^{T} y\right| \leq C^{\prime} ;\left|A_{i} x-A_{i} y\right| \leq C^{\prime} \sqrt{\log n} \cdot \sqrt{\log \min \{n, m\}} \cdot \Delta_{i}$ for all $i=1, \ldots, n_{A}$ $\left(n:=n_{A}+n_{B}\right) ;\left|B_{i} x-B_{i} y\right| \leq C^{\prime} \log \left(\frac{2}{\mu_{i}}\right) \sqrt{1 / \mu_{i}}$ for all $i=1, \ldots, n_{B}$.

- Tail bounds: For all $\lambda \geq 0$ and all $i$ :

- $\operatorname{Pr}\left[\left|A_{i} x-A_{i} y\right|>\lambda \cdot C^{\prime} \sqrt{\log \min \{n, m\}} \cdot \Delta_{i}\right] \leq 2 \cdot 2^{-\lambda^{2}}$

$-\operatorname{Pr}\left[\left|B_{i} x-B_{i} y\right|>\lambda \cdot C^{\prime} / \sqrt{\mu_{i}}\right] \leq 2 \cdot 2^{-\lambda}$.

Proof. Again we can append $c$ as an additional row to $B$, hence we ignore the objective function from now on. As described in the proof of Theorem 6 , we can assume that $m \leq n$ and that entries of $x$ have a finite dyadic expansion with $K$ bits. We perform the following algorithm:

(1) FOR $k:=K, K-1, \ldots, 1 \mathrm{DO}$

(2) $J:=\left\{j \in\{1, \ldots, m\} \mid x_{j}\right.$ 's $k$ th bit is 1$\}$

(3) Repeat computing $\chi^{(k)}: J \rightarrow\{ \pm 1\}$ according to Theorem 11 until $\chi^{(k)}$ is good, i.e. until

- $\left|A_{i} \chi^{(k)}\right| \leq C^{\prime} \cdot \sqrt{\log n} \cdot \sqrt{\log m} \cdot \Delta_{i}$ for all $i=1, \ldots, n_{A}$

- $\left|B_{i} \chi^{(k)}\right| \leq C^{\prime} \log \left(\frac{2}{\mu_{i}}\right) / \sqrt{\mu_{i}}$ for all $i=1, \ldots, n_{B}$

(4) Update $x:=x+\left(\frac{1}{2}\right)^{k} \chi^{(k)}$

Let $y$ be the integral vector obtained at the end. For $C^{\prime}$ large enough, by Theorem[1] each run to compute coloring $\chi^{(k)}$ has $\operatorname{Pr}\left[\left|A_{i} \chi\right|>C^{\prime} \sqrt{\log n} \cdot \sqrt{\log m} \cdot \Delta_{i}\right] \leq \frac{1}{4 n}$ and $\operatorname{Pr}\left[\left|B_{i} \chi\right|>C^{\prime} \log \left(\frac{2}{\mu_{i}}\right) / \sqrt{\mu_{i}}\right] \leq \frac{1}{4} \mu_{i}$. By the union bound, each run of (3) is good with probability at least $\frac{1}{2}$. By Equation (4) concerning conditional 
probabilities, this only worsens the tail bounds provided by Theorem 11 for $B_{i} \chi^{(k)}$ and $A_{i} \chi^{(k)}$ by a factor of at most 2. In any case we have a guarantee that

$$
\left|A_{i} x-A_{i} y\right| \leq \sum_{k \geq 1}\left(\frac{1}{2}\right)^{k}\left|A_{i} \chi^{(k)}\right| \leq \sum_{k \geq 1}\left(\frac{1}{2}\right)^{k} \cdot C^{\prime} \sqrt{\log n \cdot \log m} \cdot \Delta_{i}=C^{\prime} \sqrt{\log n \cdot \log m} \cdot \Delta_{i} .
$$

and analogously $\left|B_{i} x-B_{i} y\right| \leq C^{\prime} \log \left(\frac{2}{\mu_{i}}\right) / \sqrt{\mu_{i}}$. The algorithm behind Theorem 11 is fully symmetric, i.e. $E\left[A \chi^{(k)}\right]=\mathbf{0}$ for all $k$, hence $E[A y]=A x$ (and similar $E[B y]=B x$ ). It remains to prove the tail bounds. We may assume that $\lambda \geq 1$, since otherwise, the desired probabilities $2 \cdot 2^{-\lambda}$ and $2 \cdot 2^{-\lambda^{2}}$ exceed 1 anyway.

Note that if $\left|B_{i} \chi^{(k)}\right| \leq \frac{(\lambda+k) C^{\prime}}{4 \sqrt{\mu_{i}}}$ holds for all $k$, then

$$
\left|B_{i} x-B_{i} y\right|=\left|\sum_{k \geq 1}\left(\frac{1}{2}\right)^{k} B_{i} \chi^{(k)}\right| \leq \sum_{k \geq 1}\left(\frac{1}{2}\right)^{k} \frac{C^{\prime} \cdot(\lambda+k)}{4 \sqrt{\mu_{i}}} \leq \lambda \frac{C^{\prime}}{\sqrt{\mu_{i}}}
$$

since $\sum_{k \geq 1}\left(\frac{1}{2}\right)^{k} \leq 2, \sum_{k \geq 1}\left(\frac{1}{2}\right)^{k} k \leq 2$ and $\lambda \geq 1$. Hence we can use the bound

$$
\operatorname{Pr}\left[\left|B_{i} x-B_{i} y\right|>\lambda \cdot \frac{C^{\prime}}{\sqrt{\mu_{i}}}\right] \leq \sum_{k \geq 1} \operatorname{Pr}\left[\left|B_{i} \chi^{(k)}\right|>\frac{(\lambda+k) C^{\prime}}{4 C} \frac{C}{\sqrt{\mu_{i}}}\right] \stackrel{\text { Thm. }}{\leq} \sum_{k \geq 1} 320 \cdot 2^{-\frac{(\lambda+k) C^{\prime}}{24 C}} \leq 2 \cdot 2^{-\lambda}
$$

for $C^{\prime}$ large enough. Similarly

$\operatorname{Pr}\left[\left|A_{i} x-A_{i} y\right|>\lambda \cdot C^{\prime} \sqrt{\log m} \cdot \Delta_{i}\right] \leq \sum_{k \geq 1} \operatorname{Pr}\left[\left|A_{i} \chi^{(k)}\right|>\frac{(\lambda+k) C^{\prime}}{4 C} C \sqrt{\log m} \Delta_{i}\right] \stackrel{\operatorname{Thm} \cdot[1]}{\leq} \sum_{k \geq 1} 8 e^{-\frac{(\lambda+k)^{2} C^{\prime 2}}{32 C}} \leq 2 \cdot 2^{-\lambda^{2}}$

again for $C^{\prime}$ large enough and $\lambda \geq 1$.

\section{B How to solve the LP relaxations}

All linear programs for which we provided rounding procedures were of the form $\min \left\{c^{T} x \mid \sum_{S \in S} x_{S} \mathbf{1}_{S}=\right.$ $\mathbf{1}, x \geq \mathbf{0}$ \}, i.e. they all have an exponential number of variables. So, we should explain how such programs can be solved. In fact, the first polynomial time algorithm was proposed by [KK82] in the case of BIN PACKING. Their approach solves the dual $\max \left\{\sum_{i=1}^{n} y_{i} \mid \sum_{i \in S} y_{i} \leq c_{S} \forall S \in \boldsymbol{S}\right\}$ up to an arbitrarily small additive error using the Grötschel-Lovász-Schrijver variant of the Ellipsoid method [GLS81]. The error term cannot be avoided, since a PARTITION instance could be decided by inspecting whether $O P T_{f} \leq$ 2 or not. The only additional prerequisite for the Karmarkar-Karp algorithm is an FPTAS for the dual separation problem (i.e. given dual prices $y_{1}, \ldots, y_{n} \geq 0$, find a $(1-\varepsilon)$-approximation to $\max \left\{\frac{1}{c_{S}} \sum_{i \in S} y_{i} \mid\right.$ $S \in \boldsymbol{S}\}$ ). Note that the same result is implied by the framework of Plotkin, Shmoys and Tardos [PST95] without using general LP solvers.

It follows implicitly from both papers [KK82, PST95] that for any set family $S \subseteq 2^{[n]}$ that admits an FPTAS for the dual separation problem, the corresponding column-based LP can be solved within an arbitrarily small additive error.

However, we are not aware of an explicit proof of this fact in the literature. Hence, to be self-contained we provide all the details here. Our focus lies on giving a short and painless analysis, rather than giving the best bounds on the running time. Our starting point is the following theorem from [PST95] (paraphrased to make it self-contained). 
Theorem 17 (Plotkin, Shmoys, Tardos [PST95]). Let $A \in \mathbb{R}_{\geq 0}^{n \times m}$ be a matrix and $P \subseteq \mathbb{R}_{\geq 0}^{m}$ be a convex set. Given $0<\varepsilon<1, n \in \mathbb{N}, b \in \mathbb{Q}_{>0}^{n}, \rho \geq \max _{i=1, \ldots, n} \max _{x \in P} \frac{\vec{A}_{i} x}{b_{i}}$ as input. Then there exists an algorithm COVER which either computes an $x \in P$ with $A x \geq(1-\varepsilon) b$ or asserts that there is no $x \in P$ with $A x \geq b$. This algorithm calls $K:=O\left(n+\rho \log ^{2}(n)+\frac{\rho}{\varepsilon^{2}} \log \left(\frac{n}{\varepsilon}\right)\right)$ many times the following oracle (with $\varepsilon^{\prime} \in\{\varepsilon / 2,1 / 6\}$ )

Subroutine: Given $0<\varepsilon^{\prime}<1, y \in \mathbb{R}_{+}^{n}$ as input. Find a $\tilde{x} \in P$ such that $y^{T} A \tilde{x} \geq\left(1-\varepsilon^{\prime}\right) \max \left\{y^{T} A x \mid\right.$ $x \in P\}$.

Assuming that for any $\tilde{x}$, the vector $A \tilde{x}$ can be evaluated in time $O(n)$, the additional running time of COVER is $O(K \cdot n)$.

On an intuitive level, the algorithm of [PST95] maintains at any iteration some vector $x \in P$. Then for every element $i \in[n]$ one defines certain dual prizes $y_{i}$ which are decreasing in $\frac{A_{i} x}{b_{i}}$. In other words, uncovered elements will receive a high dual price $y_{i}$; covered ones receive a low price. Then one computes a vector $\tilde{x}$ which (approximately) maximizes the dual prices, meaning that $\tilde{x}$ has a large incentive to cover elements $i$ with $A_{i} x \ll b_{i}$. Then one replaces $x$ by a convex combination of $x$ and $\tilde{x}$ and iterates.

In the following we show how Theorem 17 can be used to solve (3).

Theorem 18. Let $S \subseteq 2^{[n]}$ be a family of sets with cost function $\left.\left.c: S \rightarrow\right] 0,1\right]$ (assume $c(S)$ can be evaluated in time $O(n)$ ) such that for any $y \in \mathbb{Q}_{+}^{n}$ given as input, one can find an $S^{*} \in S$ with $\sum_{i \in S^{*}} y_{i} \geq(1-\varepsilon)$. $\max \left\{\frac{1}{c(S)} \sum_{i \in S} y_{i} \mid S \in S\right\}$ in time $T(n, \varepsilon)$. Then for any given $n / 2 \geq \delta>0$, one can find a basic solution $x$ of the $L P$

$$
O P T_{f}=\min \left\{c^{T} x \mid \sum_{S \in S} x_{S} \mathbf{1}_{S} \geq \mathbf{1}, x \geq \mathbf{0}\right\}
$$

of $\operatorname{cost} c^{T} x \leq O P T_{f}+\delta$ in time $O\left(\frac{n^{4}}{\delta^{2}} \ln \left(\frac{n}{\delta}\right)\right) \cdot\left(\frac{1}{\delta} T(n, \Omega(\delta / n))+n^{2}\right)$.

Proof. Since no set costs more than 1 , one has $O P T_{f} \leq n$. By trying out $O(n / \delta)$ values, we may assume to know a value $r$ with $O P T_{f} \leq r \leq O P T_{f}+\frac{\delta}{2}$. We define $P=\left\{x \in \mathbb{R}_{\geq 0}^{S} \mid \sum_{S \in S} c_{S} x_{S}=r\right\}$ and a matrix 9 $A \in\{0,1\}^{n \times S}$ by

$$
A_{i S}= \begin{cases}1 & i \in S \\ 0 & \text { otherwise }\end{cases}
$$

as well as $b=(1, \ldots, 1)$. We choose

$$
\rho:=n \geq r \geq \max _{i=1, \ldots, m} \max _{x \in P} \frac{A_{i} x}{b_{i}}
$$

and $\varepsilon:=\frac{\delta}{4 n}$. The next step is to design the Subroutine. Hence, let a vector of dual prices $y \in \mathbb{Q}_{\geq 0}^{n}$ and a parameter $\varepsilon^{\prime}>0$ be given as input. Then we compute a set $S^{*} \in S$ with $\sum_{i \in S^{*}} y_{i} \geq\left(1-\varepsilon^{\prime}\right) \cdot \max \left\{\frac{1}{c_{S}} \sum_{i \in S} y_{i} \mid\right.$ $S \in S\}$ in time $T\left(n, \varepsilon^{\prime}\right)$. Observe that the vertices of $P$ are of the form $\frac{r}{c_{S}} e_{S}$ and

$$
y^{T} A\left(\frac{r}{c_{S}} e_{S}\right)=\frac{r}{c_{S}} \sum_{i=1}^{n} y_{i} A_{i S}=\frac{r}{c_{S}} \sum_{i \in S} y_{i}
$$

hence, the vector $\tilde{x}:=\frac{r}{c_{S}} \cdot e_{S^{*}}$ is the desired $\left(1-\varepsilon^{\prime}\right)$-approximation for $\max \left\{y^{T} A x \mid x \in P\right\}$.

Applying Theorem[17yields a vector $x \in P$ with $A x \geq(1-\varepsilon)$ 1. Hence the slightly scaled vector $x^{\prime}=$ $\frac{x}{1-\varepsilon}$ is feasible and has cost $\frac{r}{1-\varepsilon} \leq\left(O P T_{f}+\frac{\delta}{2}\right) \cdot(1+2 \varepsilon) \leq O P T_{f}+\delta$. To turn $x^{\prime}$ into a basic solution,

\footnotetext{
${ }^{9} A$ and $P$ are defined, but not explicitly computed.
} 
we consider $x^{\prime \prime}$ with $x_{S}^{\prime \prime}=x_{S}^{\prime}>0$ for precisely $n+1$ many sets (and $x_{S}^{\prime \prime}=0$ otherwise). Then by Gauss elimination we find a $x^{\prime \prime \prime} \geq \mathbf{0}$ with $A x^{\prime \prime \prime}=A x^{\prime \prime},\left|\operatorname{supp}\left(x^{\prime \prime \prime}\right)\right| \leq n$ and $c^{T} x^{\prime \prime \prime} \leq c^{T} x^{\prime \prime}$ in time $O\left(n^{3}\right)$ and replace the corresponding values in $x^{\prime}$ by those in $x^{\prime \prime \prime}$. After iteration this at most $K$ times, we obtain the desired basic solution. The total running time is

$$
\frac{n}{\delta} \cdot K \cdot T(n, \Omega(\varepsilon))+O\left(K \cdot n^{3}\right) \leq O\left(\frac{n^{4}}{\delta^{2}} \ln \left(\frac{n}{\delta}\right)\right) \cdot\left(\frac{1}{\delta} T(n, \Omega(\delta / n))+n^{2}\right)
$$

since SUBROUTINE was called at most $K=O\left(\frac{n^{3}}{\delta^{2}} \ln \left(\frac{n}{\delta}\right)\right)$ times.

Here the running times are w.r.t. the RAM model, were any arithmetic operation accounts with unit cost.

\section{Applications for considered problems}

In order to solve the considered LPs up to any additive error term, it suffices to provide an FPTAS for each of the corresponding dual separation problems.

- BIn PACKIng : The dual separation problem is $\max \left\{\sum_{i \in S} y_{i} \mid \sum_{i \in S} s_{i} \leq 1\right\}$ which is known as KNAPSACK problem and admits an FPTAS in time $T(n, \varepsilon)=O\left(n / \varepsilon^{2}\right)$ (see e.g. [Vaz01]).

- Bin Packing With Rejection: We compute a $(1-\varepsilon)$-approximation $S^{*}$ to $\max \left\{\sum_{i \in S} y_{i} \mid \sum_{i=1}^{n} s_{i} \leq\right.$ $1\}$ in time $O\left(n / \varepsilon^{2}\right)$ and compare it to the values $\frac{y_{i}}{\pi_{i}}$ for $i=1, \ldots, n$ and output either $S^{*}$ or some $\{i\}$, whoever yields the largest value, hence again $T(n, \varepsilon)=O\left(n / \varepsilon^{2}\right)$.

- Train Delivery. For any $k \in\{1, \ldots, n\}$, let $S^{k}$ be a $(1-\varepsilon)$-approximate solution to $\max \left\{\sum_{i \in S} y_{i} \mid\right.$ $\left.\sum_{i \in S} s_{i} \leq 1, S \subseteq\left\{i \mid p_{i} \leq p_{k}\right\}\right\}$. We output the set $S^{k}$ maximizing $\sum_{i \in S^{k}} \frac{y_{i}}{p^{k}}$. This can be done in time $T(n, \varepsilon)=O\left(n^{2} / \varepsilon^{2}\right)$.

\section{Omitted proof for Lemma 5}

Lemma (Lemma5). Let $\alpha \in \mathbb{R}^{m}$ be a vector and $\Delta>0$. For $\lambda=\frac{\Delta}{\|\alpha\|_{2}}$,

$$
\underset{\chi \in\{ \pm 1\}^{m}}{H}\left(\left\lceil\frac{\alpha^{T} \chi}{2 \Delta}\right]\right) \leq G(\lambda):= \begin{cases}9 e^{-\lambda^{2} / 5} & \text { if } \lambda \geq 2 \\ \log _{2}(32+64 / \lambda) & \text { if } \lambda<2\end{cases}
$$

Proof. We distinguish 2 cases. Case $\lambda \geq 2$. Let $p_{i}:=\operatorname{Pr}[Z=i]$. Note that $X:=\alpha^{T} \chi=\sum_{j=1}^{m} \alpha_{j} \cdot \chi_{j}$ is the sum of independently distributed random variables $\chi_{j} \cdot \alpha_{j}= \pm\left|\alpha_{j}\right|$ with mean 0 . For $i \geq 1$,

$$
p_{i} \leq \operatorname{Pr}[X \geq(2 i-1) \Delta] \stackrel{\text { Lem. [1 }}{\leq} 2 e^{-\frac{(2 i-1)^{2} \Delta^{2}}{2\|\alpha\|_{2}^{2}}}=2 e^{-(2 i-1)^{2} \lambda^{2} / 2} \stackrel{\lambda \geq 2}{\leq} e^{-(2 i-1)^{2} \lambda^{2} / 4}
$$

The entropy, stemming from $i \geq 1$ is fairly small, namely

$$
\sum_{i \geq 1} p_{i} \log _{2}\left(\frac{1}{p_{i}}\right) \leq \sum_{i \geq 1} e^{-(2 i-1)^{2} \lambda^{2} / 4} \cdot \log _{2}\left(\frac{1}{e^{-(2 i-1)^{2} \lambda^{2} / 4}}\right)=\sum_{i \geq 1} e^{-(2 i-1)^{2} \lambda^{2} / 4} \cdot \frac{(2 i-1)^{2} \lambda^{2}}{4 \cdot \ln (2)} \leq 3 \cdot e^{-\lambda^{2} / 5}
$$


Here we use that $p_{i} \leq \frac{1}{e}$ and $x \cdot \log _{2}\left(\frac{1}{x}\right)$ is monotone increasing for $x \in\left[0, \frac{1}{e}\right]$. Furthermore $p_{0} \geq 1-\operatorname{Pr}[|X| \geq$ $\Delta] \geq 1-2 e^{-\lambda^{2} / 2}$, hence the event $\{Z=0\}$ is so likely that it also does not contribute much entropy.

$$
p_{0} \log \left(\frac{1}{p_{0}}\right) \leq 2 \cdot\left(1-p_{0}\right) \leq 4 e^{-\lambda^{2} / 2} \leq 2 e^{-\lambda^{2} / 5}
$$

Adding up also the entropy for $i<0$, we obtain $H(Z) \leq 8 \cdot e^{-\lambda^{2} / 5}$.

Case $\lambda<2$. Define $L:=\left\lceil\frac{2}{\lambda}\right\rceil>1$ and $\Delta^{\prime}:=\Delta \cdot L$. Then we can express $Z=L \cdot\left\lceil\frac{X}{2 L \Delta}\right\rfloor+Z^{\prime \prime}$ such that $Z^{\prime \prime}$ attains just $L$ different values (and hence $H\left(Z^{\prime \prime}\right) \leq \log _{2}(L)$ ). Let $\lambda^{\prime}:=\Delta^{\prime} /\|\alpha\|_{2} \geq 2$, then

$$
H(Z) \stackrel{(*)}{\leq} H\left(\left[\frac{X}{2 \Delta^{\prime}}\right\rfloor\right)+H\left(Z^{\prime \prime}\right) \stackrel{(* *)}{\leq} 9 e^{-\lambda^{\prime 2} / 5}+\log _{2}(L) \leq \underbrace{9 \cdot e^{-4 / 5}}_{<5}+\log _{2}\left(\frac{2}{\lambda}+1\right)
$$

In $(*)$, we use the subadditivity of the entropy function. In $(* *)$ we use that $H\left(\left\lceil\frac{X}{2 \Delta^{\prime}}\right\rfloor\right)$ can be bounded by case (1). 